\title{
Centrosome guides spatial activation of Rac to control cell polarization and directed cell migration
}

\author{
Hung-Wei Cheng ${ }^{1, \star}$, Cheng-Te Hsiao ${ }^{2, *}$, Yin-Quan Chen ${ }^{3,4} \mathbb{0}$, Chi-Ming Huang $\mathbb{D}^{1}$, Seng-I Chan ${ }^{1}$, Arthur Chiou ${ }^{4}$, \\ Jean-Cheng Kuo ${ }^{1,3}$ (1)
}

\begin{abstract}
Directed cell migration requires centrosome-mediated cell polarization and dynamical control of focal adhesions (FAs). To examine how FAs cooperate with centrosomes for directed cell migration, we used centrosome-deficient cells and found that loss of centrosomes enhanced the formation of acentrosomal microtubules, which failed to form polarized structures in wound-edge cells. In acentrosomal cells, we detected higher levels of Rac1guanine nucleotide exchange factor TRIO (Triple Functional Domain Protein) on microtubules and FAs. Acentrosomal microtubules deliver TRIO to FAs for Rac1 regulation. Indeed, centrosome disruption induced excessive Rac1 activation around the cell periphery via TRIO, causing rapid FA turnover, a disorganized actin meshwork, randomly protruding lamellipodia, and loss of cell polarity. This study reveals the importance of centrosomes to balance the assembly of centrosomal and acentrosomal microtubules and to deliver microtubule-associated TRIO proteins to FAs at the cell front for proper spatial activation of Rac1, FA turnover, lamillipodial protrusion, and cell polarization, thereby allowing directed cell migration.
\end{abstract}

DOI 10.26508/Isa.201800135 | Received 19 July 2018 | Revised 27 January 2019 | Accepted 28 January 2019 | Published online 8 February 2019

\section{Introduction}

Cell migration is a critical process in the development and maintenance of multicellular organisms and is involved in many important cell processes, including tissue formation during embryogenesis, wound healing, and various types of immune response (Franz et al, 2002). In many cases, the orchestrated movement of a cell is required to allow migration to a specific location or locations; this is a complex and highly coordinated process driven by various cell-scale dynamic macromolecular ensembles, one of which is the cytoskeleton system. Initially, migrating cells become polarized toward the direction of movement, and this occurs via reorientation of the microtubule-organizing center (MTOC) including the centrosome and the Golgi apparatus (Nobes \& Hall, 1999; Etienne-Manneville \& Hall, 2001); this results in the assembly of microtubules at the front of the cell and promotion of the dynamic polymerization of actin to extend a membrane protrusion. Subsequently, the protruding membrane adheres to the ECM via the formation of a number of cellular adhesive organelles, namely, the focal adhesions (FAs). FAs are connected to the actin cytoskeleton and transduce contractile force along the bundles of actin filaments (the stress fibers), which acts on the ECM; the result is a maturation process that pulls the cell body forward. Finally, FA disassembly occurs, and this is accompanied by myosin II-mediated contractile forces that pull the trailing edge of the cell away from the ECM (Huttenlocher et al, 1996; Lauffenburger \& Horwitz, 1996; Webb et al, 2002; Ridley et al, 2003). The dynamics of the microtubules, the various actin networks, and the FAs need to be orchestrated in a precise spatial and temporal order to bring about directed cell migration (Gupton \& Waterman-Storer, 2006). Any errors that occur during the process of cell migration can result in a range of serious consequences, including intellectual disability, vascular disease, tumor formation, and metastasis (Franz et al, 2002).

FAs are the organelles that allow transient ECM attachment at the cell membrane. FAs start to form when their central component, the integrin receptor, is activated by engagement with the ECM. This is subsequently followed by the recruitment of a series of FAassociated proteins that are able to connect to the actin cytoskeleton (Jockusch et al, 1995; Schwartz et al, 1995; Burridge et al, 1988; Hynes, 2002; Zaidel-Bar et al, 2007; Zaidel-Bar \& Geiger, 2010). A subset of nascent FAs (new-born FAs) grows and changes protein composition in a process called FA maturation. Mature FAs then either stabilize or begin to disassemble underneath the cell body and at the rear of the cell. Spatial and temporal control of FA turnover brings about dynamic remodeling of the abundance of various groups of proteins in FAs (Kuo et al, 2011), and these changes allow cells to respond to extracellular cues and to transduce specific signals that bring about the modulation of the cytoskeletal system needed for coordinated and productive cell movement (Wu et al, 2015; Yu et al, 2015). The molecular

${ }^{1}$ Institute of Biochemistry and Molecular Biology, National Yang-Ming University, Taipei, Taiwan ${ }^{2}$ Institute of Biological Chemistry, Academia Sinica, Taipei, Taiwan ${ }^{3}$ Cancer Progression Research Center, National Yang-Ming University, Taipei, Taiwan ${ }^{4}$ Institute of Biophotonics, National Yang-Ming University, Taipei, Taiwan 
mechanisms underlying the regulation of FA turnover are complex, and dynamic microtubules are starting to reveal its importance (Stehbens \& Wittmann, 2012). It has been reported that microtubules that are able to stochastically undergo switching between phases of growth and phases of shortening probably influence FA turnover by locally modulating Rho GTPase signaling (WatermanStorer et al, 1999; Ezratty et al, 2005; Chang et al, 2008; Rooney et al, 2010) and clathrin-dependent (Ezratty et al, 2005, 2009; Nishimura \& Kaibuchi, 2007; Chao \& Kunz, 2009) and clathrin-independent endocytosis (Echarri \& Del Pozo, 2006). Thus, microtubules are central to spatially controlled FA dynamics and this, in turn, is involved in modulating the outcome of downstream integrin engagement and eventually control of cell migration.

Microtubules are dynamically orientated by the centrosome and other MTOCs, and this results in centrosomal and acentrosomal networks, respectively. The molecular mechanisms by which centrosomal and acentrosomal microtubules regulate directional migration remain unknown up to present, although the centrosome has long been recognized as defining the front of a cell (Wakida et al, 2010; Zhang \& Wang, 2017). To address the above question, we have used three types of human retinal pigment epithelial (RPE) cells, control cells (RPEp53-/- cells [Bazzi \& Anderson, 2014]) and acentriolar cells (RPEp53 $3^{-1-} \mathrm{SAS6}^{-/-}$cells [Wang et al, 2015] and RPEp53 ${ }^{-/-} \mathrm{STIL}^{-1-}$ cells [Chen et al, 2017]) to characterize the compositional changes in FAs that occur in response to centrosome disruption. We found that centrosome disruption enhances the assembly of acentrosomal microtubules and these are associated with and involved in the delivery of the Rac1 guanine nucleotide exchange factor (GEF), TRIO (Triple Functional Domain Protein), to FAs; this then promotes excessive Rac1 activation and brings about changes in lamellipodial protrusion, which results in differences in FA turnover. The present study addresses the roles of the centrosome in restricting the distribution of acentrosomal microtubules, which in turn affects the activation of Rac1 at the front of a migrating cell.

\section{Results}

\section{Organization by the centrosome mediates cell polarization and guides directed cell migration}

Previous studies have demonstrated that the cellular position of the centrosome is indicative of cell polarization during directed cell migration (Wakida et al, 2010; Zhang \& Wang, 2017). To investigate if assembly of the centrosome mediates cell polarization and guides directed cell migration, we examined the effect in retinal pigment epithelial (RPE) cells of disrupting centriole formation (centrosome assembly). Acentriolar cells are not viable in the presence of the p53 gene (Bazzi \& Anderson, 2014); therefore, RPEp53/-- cells, which are null for the p53 gene, were used for this part of the study. SAS6 and STIL are the main components in daughter centriole involved in centriole duplication (Leidel et al, 2005; David et al, 2014; Kim et al, 2014; Arquint \& Nigg, 2016); thus, the acentriolar cell lines (RPEp53 $^{-1-} \mathrm{SAS6}^{-1-}$ [Wang et al, 2015] and RPEp53 ${ }^{-1-} \mathrm{STIL}^{-1-}$ cells [Chen et al, 2017]) were generated by knocking out SAS6 and STIL separately, using CRISPR/Cas9 technology. Immunoblotting of total cell lysates validated that SAS6 and STIL had been knocked out in the RPEp53 ${ }^{-/-} \mathrm{SAS6}^{-/-}$and RPEp53 ${ }^{-/-} \mathrm{STIL}^{-/-}$cells, respectively (Fig 1A). After knockout, the expression of $y$-tubulin, a main component of the pericentriolar material that is involved in mediating microtubule nucleation, was found to still have similar levels in $\mathrm{RPEp53}^{-/-}, \mathrm{RPEp}^{53^{-/-} \mathrm{SAS6} 6^{-/-} \text {, and RPEp53 }}{ }^{-/-} \mathrm{STIL}^{-/-}$cells (Fig 1A). Immunofluorescence microscopy analysis revealed that there was accumulation of $y$-tubulin at the centrosome in RPEp53-/- cells, but

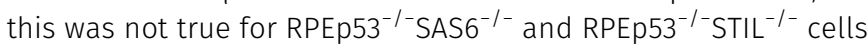
(Fig 1B). Therefore, knockout of either SAS6 or STIL only disrupts the assembly of centrosome but does not seen to affect the expression of pericentriolar material components.

We next determined if an intact centrosome structure is able to modulate directed cell migration; this was assessed by woundhealing migration assay. We measured the percentage of wound closure over 705 min of migration period and found that cells that were null for SAS6 or STIL showed a marked acceleration in wound closure (Fig 1C). We further tracked the trajectory of single individual cells at wound edge over the same period by tracing the center of their nuclei and found that RPEp53 $3^{-1-} \mathrm{SAS6} 6^{-/-}$and RPEp53 ${ }^{-1-} \mathrm{STIL}^{-1-}$ cells had a much faster migration speed, but poor directional persistence, compared with the control cells (RPEp53-/-) (Fig 1D). These effects could be visualized via displays of representative cell migration paths using window plots; these showed

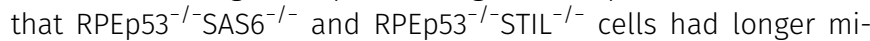
gration path and changed direction much more frequently than the control cells (Fig 1E). The increased migration path and the reduced directional persistence suggest that the loss of an intact centrosome enhances cell motility but renders these cells unable to maintain a stable direction during targeted migration. The polarization of wound-edge cells, when judged by the reorientation of their Golgi apparatus in the direction of wound, revealed that a signifi-

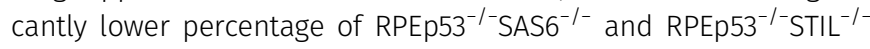
cells in the front row of cells had a polarized Golgi apparatus (Fig 1F). Closer examination of the cells at the wound edge revealed that $\mathrm{RPEp}^{-1-}$ cells displayed a polarized phenotype that included microtubules elongation to the tip of protrusion, whereas on the other hand, RPEp53 $3^{-/-} \mathrm{SAS6}^{-/-}$and RPEp53 ${ }^{-/-} \mathrm{STIL}^{-/-}$cells showed a scattered microtubule distribution and a decreased percentage of cells with polarized microtubules (Fig 1G). These observations indicate that an intact centrosome does regulate wound-healing migration and it does this by controlling cell polarization and cell motility during directed cell migration.

\section{Loss of the centrosome enhances the assembly of acentrosomal microtubules}

To understand how an intact centrosome controls directed cell migration, we focused on microtubules. We first stained various cells with GOLPH2 (Golgi complex marker) and $\alpha$-tubulin (a microtubules marker) to visualize the changes in the association of microtubules with the Golgi complex in RPEp53-/-SAS6 ${ }^{-/-}$and RPEp53 $3^{-/-} \mathrm{STIL}^{-1-}$ cells compared with control cells (Fig 2A). The results support the notion that the Golgi apparatus also serves as an MTOC and is involved in acentrosomal microtubule nucleation and stabilization (Chabin-Brion et al, 2001; Efimov et al, 2007; Zhu \&

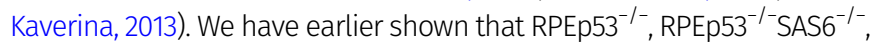


䖪踏 Life Science Alliance
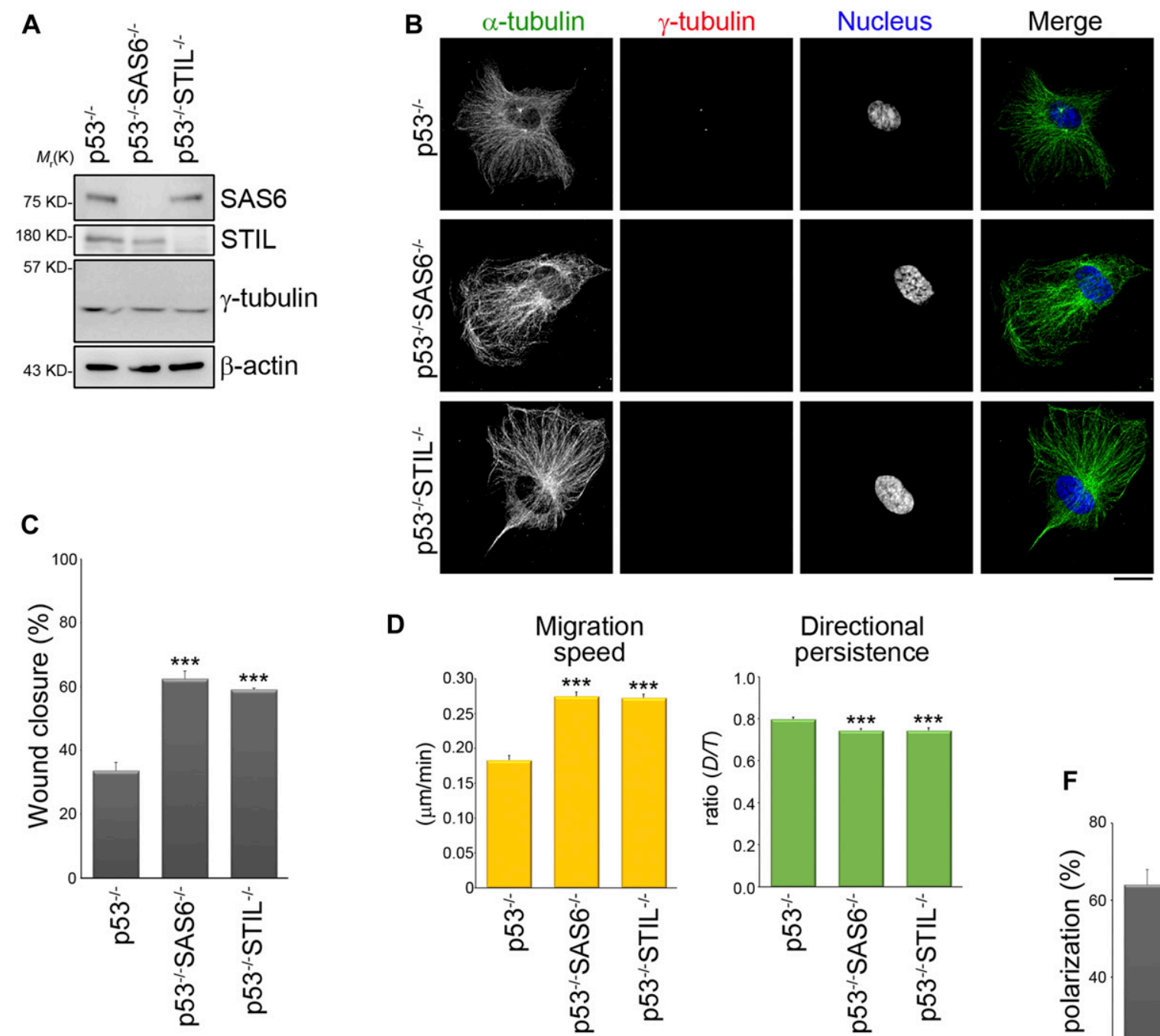

D

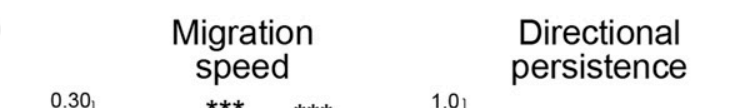

\begin{abstract}
persistence
\end{abstract}
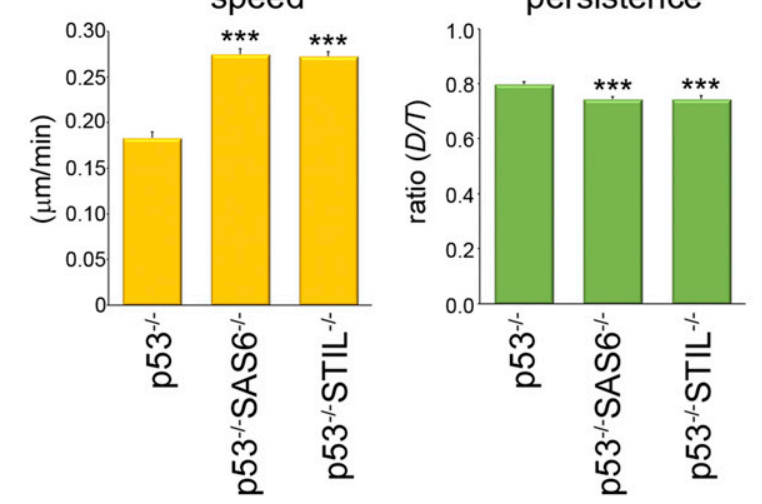

E

$$
\text { p53-- }
$$

p53--SAS6 ${ }^{-1-}$

$$
\text { p53 } 3^{--S} \text { TIL }^{-/-}
$$
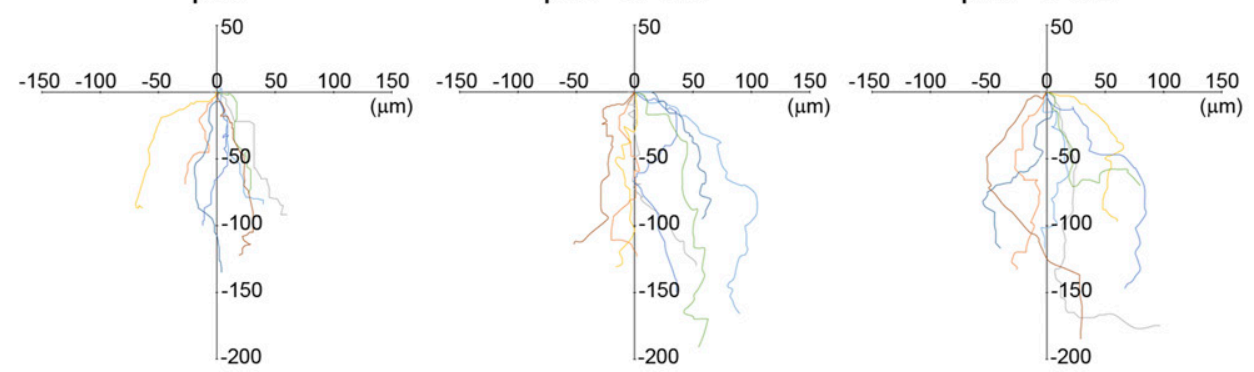

G

p53-

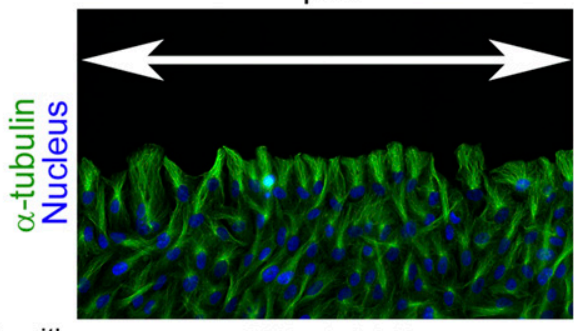

$73 \%+/-0.043$ p53 $3^{--S A S 6} 6^{--}$

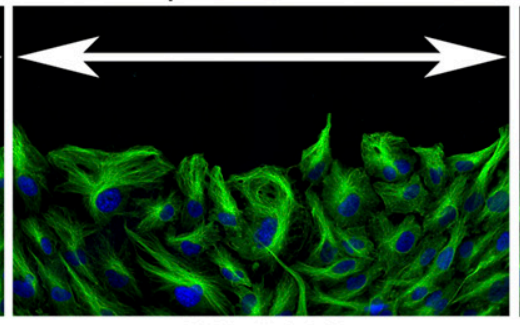

$33 \%+/-0.029$
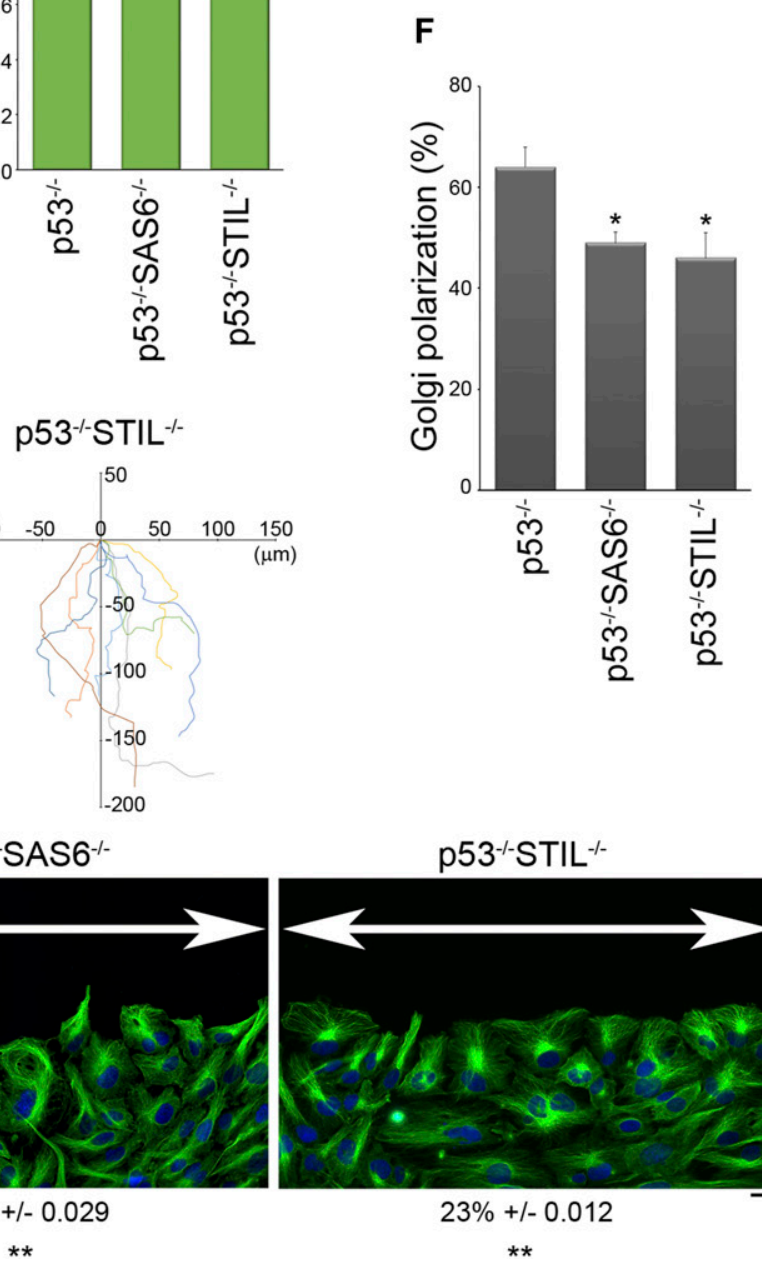

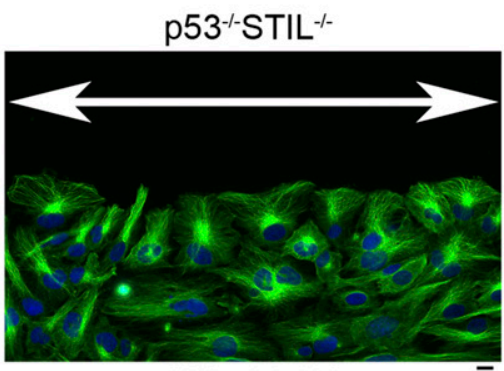

$23 \%+/-0.012$ 
and RPEp53-/- $\mathrm{STIL}^{-/-}$cells expressed similar amount of $\alpha$-tubulin in their total cell lysates (Fig 2B) and, therefore, we were able to further characterize whether the centrosome modulated the total amount of polymerized microtubules present in these cells. The polymerized microtubule-containing fraction (pellets; insoluble fraction) and the free tubulin fraction (supernatant; soluble fraction) were isolated from control cells (RPEp53 ${ }^{-/-}$cells) and acentrosomal cells (RPEp53 ${ }^{-1-} \mathrm{SAS6}^{-/-}$ and RPEp53-/-STIL ${ }^{-/-}$cells) using a previously published microtubule isolation method (Sloboda, 2015; see the Materials and Methods section). An actin-binding protein, non-muscle myosin IIA (NMIIA), was mainly detected in soluble fraction by Western blotting (Fig 2B), which confirmed that this microtubule isolation method was able to separate non-microtubule-binding proteins from the polymerized microtubule-containing fraction (insoluble fraction). We compared the abundance of $\alpha$-tubulin in polymerized microtubule-containing fraction (insoluble) and the free tubulin fraction (soluble) by Western blotting, and this revealed that acentrosomal cells contained a higher level of polymerized microtubules and that these were highly acetylated (Fig 2B). Tubulin acetylation has been demonstrated to enhance microtubule stabilization (Portran et al, 2017) and, thus, these findings indicate that loss of the centrosome increases microtubule polymerization and stabilization.

It is known that microtubule plus end dynamics influences cell migration (Waterman-Storer et al, 1999; Wittmann et al, 2003), and, therefore, we further characterized the assembly and disassembly of microtubule plus ends in control cells (RPEp53 $3^{-1-}$ cells) and in

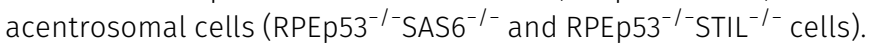
We performed live-cell imaging using fluorescently labeled EB3 (GFP-EB3), which is known to label the growing plus tips of microtubules (Fig $2 \mathrm{C}$ and Video 1-3). In the time-lapse image series showing GFP-EB3, we tracked the dynamics of the growing plus tips of the microtubules using a previously developed software package, namely, plusTip-Tracker (Matov et al, 2010). This allowed us to measure microtubule growth speed and growth persistence (growth lifetime; 1/catastrophe frequency). We then set the median values of the microtubule growth speed and the growth lifetime obtained from the pooled microtubules tracks of RPEp53/- cells as two thresholds. Based on these thresholds, the

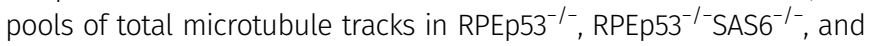
RPEp53 $3^{-1-} \mathrm{STIL}^{-1-}$ cells were classified into two pairs of subgroups, namely, "slow (red)" versus "fast (blue)" and "short-lived (yellow)" versus "long-lived (green)." In the two types of acentrosomal cells, the population of growing microtubules with slow and short-lived growth was found to be increased, which resulted in an overall trend of microtubule growth in these cells when an intact centrosome was absent (Fig 2D). The loss of a cell's centrosome, thus, significantly decreased mean microtubule growth speed and mean microtubule lifetime compared with control cells (Fig 2E). Thus, the loss of an intact centrosome causes modulation of microtubule plus end dynamics, which can be observed as slowing of microtubule assembly and a reduction in microtubule persistence. These effects can be interpreted as promotion of microtubule dynamic instability.

To characterize actin network remodeling that occurs in acentrosomal cells, the cells were immunostained to detect both F-actin (phalloidin staining) and microtubules ( $\alpha$-tubulin staining) using

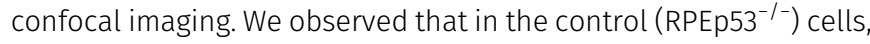
there was a dense F-actin meshwork enriched on one side of the cell's periphery that led to a front/back asymmetry distribution associated with membrane protrusions. In contrast, the acentrosomal cells had an enriched polymerized actin meshwork around the cell periphery that gave rise to multiple huge membrane protrusions (Fig 2F). We further examined the role of the centrosome in lamellipodial formation. Immunolocalization in the cells of a lamellipodia marker cortactin showed that loss of the centrosome resulted in a significant increase in the ratio of lamellipodial area to total cell area (Fig 2G). This agrees with a previous study indicating that growing microtubules govern actin network remodeling (Henty-Ridilla et al, 2016), and these findings further suggest that acentrosomal microtubules are able to enhance actin polymerization and the assembly of multiple membrane protrusions. Thus, the centrosome is able to control migrating cell polarization in a microtubule-dependent manner that involves the regulation of the distribution of front/back asymmetry with respect to membrane protrusions, and this occurs via local modulation of the formation of dense actin networks.

\section{Loss of the centrosome enhances cell adhesion}

We next investigated whether assembly of the centrosome controls cell adhesion strength. To do this, we initially examined the ad-

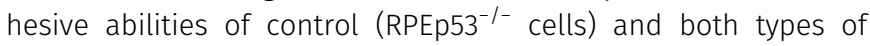

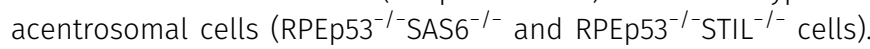
The cells were seeded on plates coated with fibronectin for $15 \mathrm{~min}$ to allow analysis of the cell spreading area (Fig 3A). The results revealed a large spreading area when acentrosomal cells were

Figure 1. The absence of the centrosome suppresses the cell polarity of a migrating cell.

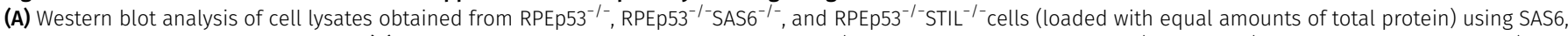
STIL, $\gamma$-tubulin, and $\beta$-actin antibodies. (B) Confocal images of immunolocalized $\alpha$-tubulin (to visualize microtubules; green), $\gamma$-tubulin (to visualize centrosome; red), and

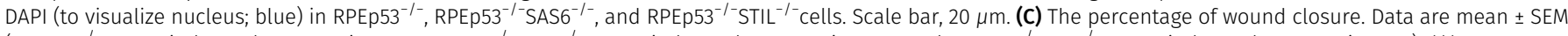

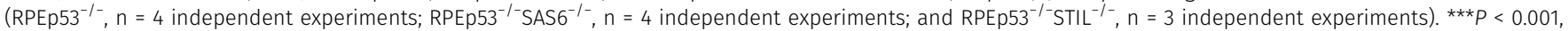
compared with RPEp53 ${ }^{-/-}$. (D) The migration parameters were calculated as described in the Materials and Methods section. Migration speed was calculated as the total length of the migration path divided by the duration of migration; directional persistence was calculated as the net migration distance divided by the total length of the migration path. Data are mean \pm SEM ( $n=100$ cells for each conditions). ${ }^{* \star *} P<0.001$, compared with RPEp53 ${ }^{-1-}$. (E) Analysis of migration trajectories. The trajectories of representative cells are plotted. The origins of migration are superimposed at $(0,0)$. (F) The percentage of Golgi reorientation. The percentage of wound-edge cells with their Golgi apparatus in the forward-facing $120^{\circ}$ sector was measured at $3 \mathrm{~h}$ after wounding. For each experiment, 100 cells were scored. Data are mean $\pm \mathrm{SEM}$ ( $\mathrm{n}=3$

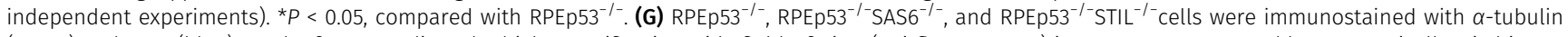
(green) and DAPI (blue) at $3 \mathrm{~h}$ after wounding. The high magnification wide field-of-view (epi-fluorescence) images were generated by automatically stitching multiple adjacent frames from a multipoint using the Nikon analysis software NIS-Elements. Arrows indicate the direction of the wound. Scale bar, $20 \mu \mathrm{m}$. The values indicate the percentage of cells with polarized microtubules. For each experiment, 100 cells were scored. Data are mean \pm SEM ( $n=3$ independent experiments). ${ }^{\star *} P<0.01$, compared with RPEp53/- 
A

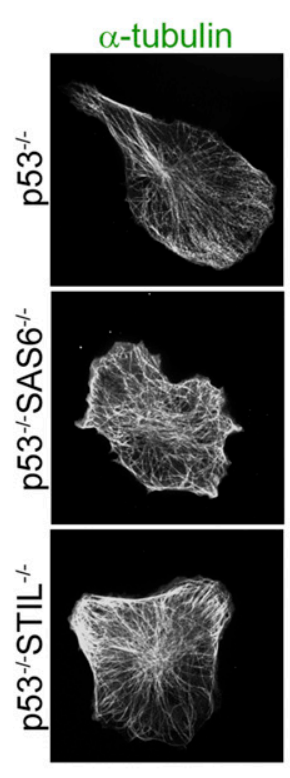

GOLPH2

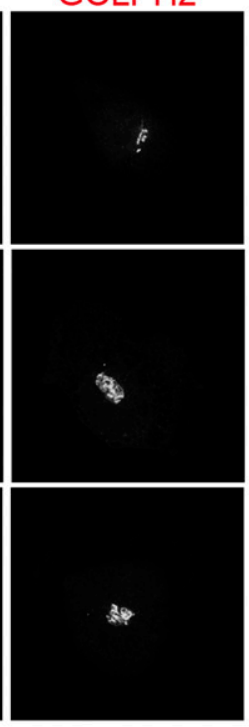

Nucleus
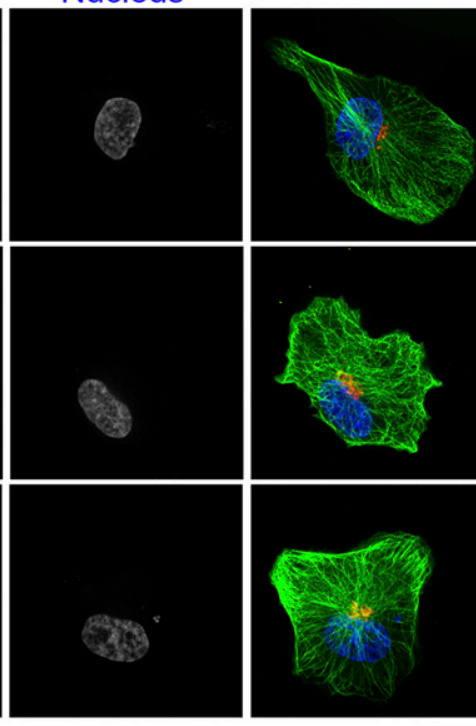

B

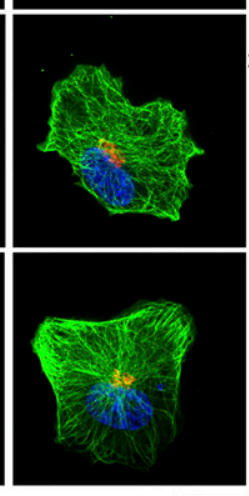

C

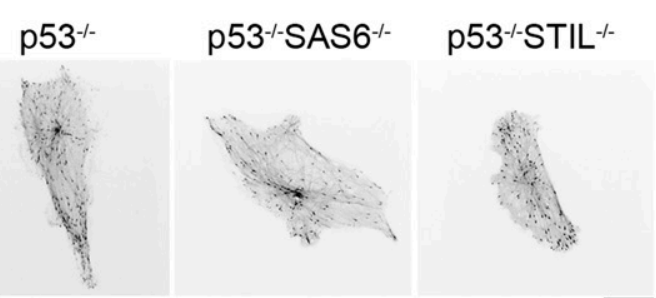

E
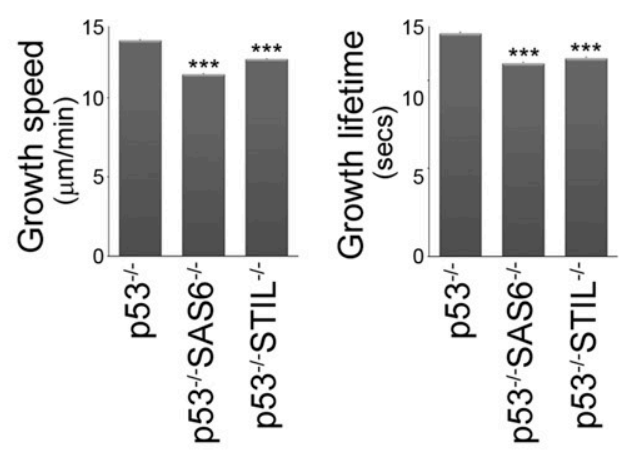

G

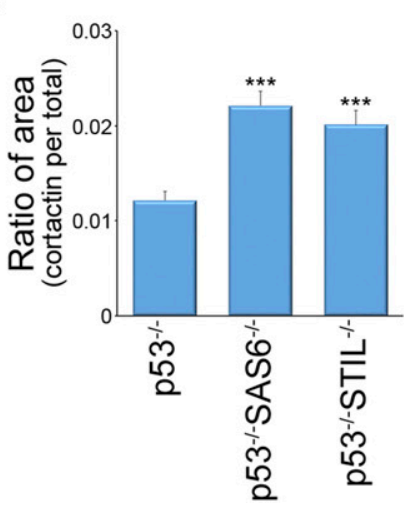

D

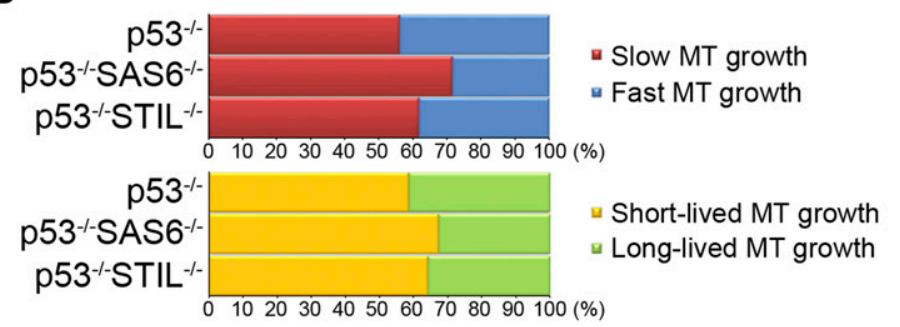

$\mathbf{F}$

F-actin

$\alpha$-tubulin

Nucleus
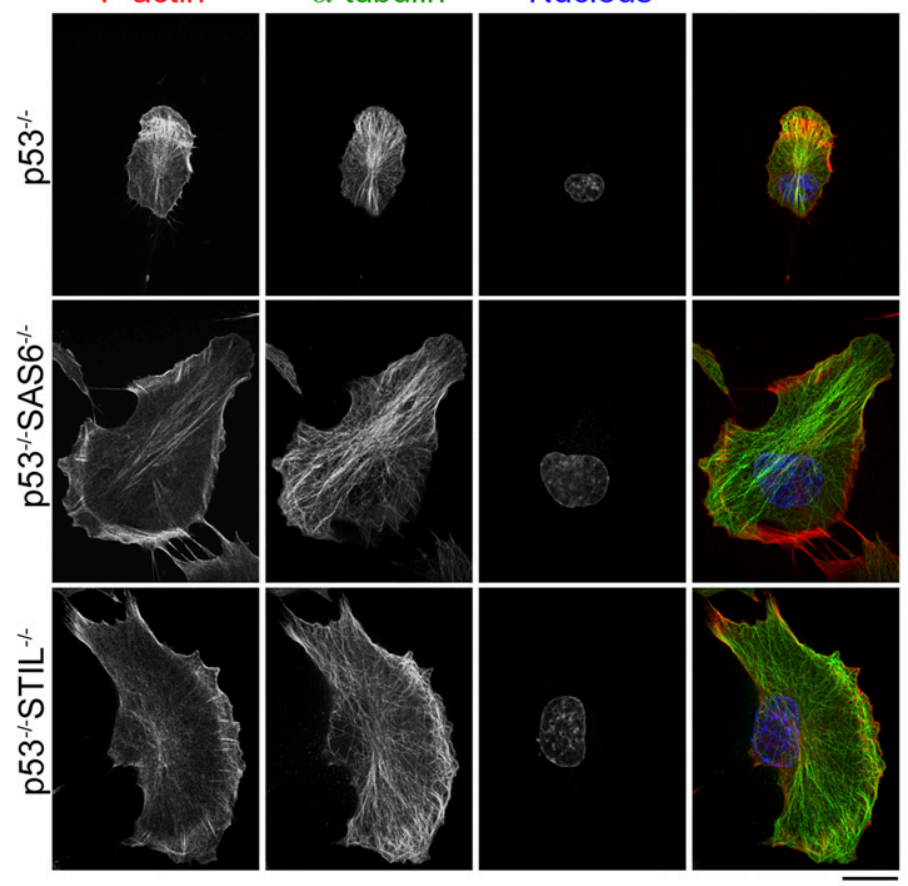
used compared with a smaller spreading area for the control cells (Fig 3B). Next, we further quantified cell adhesiveness and found that the loss of the centrosome increased cell adherence to fibronectin (Fig 3C). Thus, we were able to hypothesize that the microtubules in acentrosomal cells are involved in regulating FA formation and/or FA dynamics to enhance cell adhesion.

To investigate whether microtubules in acentrosomal cells contribute to FA dynamics in a different way to what occurs in control cells, we analyzed the size of FAs in the control and acentrosomal cells using kinetic studies of their recovery after treatment with nocodazole. The cells were incubated in a growth medium containing $10 \mu \mathrm{M}$ nocodazole overnight to stop microtubules growth and then fixed at the specific time points after perfusion with nocodazole-free medium that would induce microtubule growth. When untreated, the control condition, we found that acentrosomal cells formed more FAs (RPEp53-/- cells: 28 FAs/ cell, RPEp53 $3^{-1-}$ SAS6 $^{-1-}: \sim 111 \mathrm{FAs} /$ cell, and RPEp53 ${ }^{-/-} \mathrm{STIL}^{-/-}$cells: 135 FAs/cell), which supports the results in Fig $3 \mathrm{C}$ and indicates that cells lacking a centrosome are more adhesive. After nocodazole treatment ( 0 min washout), the cells were found to contain a larger number of big $\left(>2 \mu \mathrm{m}^{2}\right)$ and mid-sized $\left(1-2 \mu \mathrm{m}^{2}\right)$ FAs and a reduced number of small $\left(<1 \mu \mathrm{m}^{2}\right)$ FAs compared with the untreated (control) cells (Fig 3D). At 3 min after perfusion with nocodazolefree medium, we found that the acentrosomal cells showed a significant increase in the number of small FAs and a decrease in the number of big FAs compared to the RPEp53 ${ }^{-/-}$cells (Fig 3D). This suggests that loss of the centrosome promotes FA formation in a microtubule-dependent manner. Analysis of GFP-paxillin dynamics revealed that loss of the centrosome also significantly shortened FA lifetime (Fig 3E and F), especially the time needed for FA assembly and disassembly (Fig 3G). FA turnover has been shown to be regulated by dynamic microtubules attaching to FAs (Stehbens \& Wittmann, 2012); thus, we hypothesize that the enhancement in acentrosomal microtubules that is induced by centrosome disruption may deliver signals to FAs and control FA dynamics. Indeed, immunolocalization of paxillin and $\alpha$-tubulin revealed that FAs were co-localized with microtubules in both the control

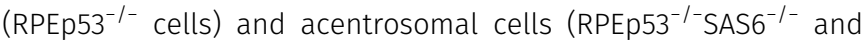
RPEp53 $3^{-1-} \mathrm{STIL}^{-1-}$ cells) (Fig 3H). Therefore, loss of the centrosome causes promotion of FA formation and increase in the turnover of FAs; this occurs most likely via specific signals delivered from the acentrosomal microtubules.

\section{Centrosome reduces lamellipodial protrusion factors in FAs}

To further characterize how FA-mediated signals are modulated by the centrosome, we analyzed the composition and abundance of proteins in FAs fractionated from control cells (RPEp53-/- cells) and

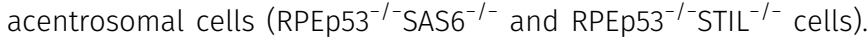
The cells were plated on fibronectin-coated plates and hypotonically shocked to separate the FA fractions using a previously described FA isolation method (Kuo et al, 2011; Kuo et al, 2012; see the Materials and Methods section); this method had been previously confirmed to preserve the native organization of the FAs. The isolated FA fractions were subject to liquid chromatography (LC)-tandem mass spectrometry (MS/MS) analysis. Following the LC-MS/MS analysis, the results were processed using a software program, Mascot, to identify the proteins present. The proteins that were identified in at least two of four replicate runs (four independent experimental runs for each condition) were included in the lists of reproducible proteins, namely, the reproducible lists. These lists indicated that there were 1,235 proteins in the FAs of RPEp53-/- cells (Table S1), 1,233 proteins in the FAs of RPEp53 $^{-1-} \mathrm{SAS6}^{-1-}$ cells (Table S2), and 1,228 proteins in the FAs of RPEp53 $3^{-1-} \mathrm{STIL}^{-1-}$ cells (Table S3).

To characterize the effect of the centrosome on the changes in abundance of FA proteins, we evaluated the relative levels of the individual FA protein isolated from the control cells (RPEp53 $3^{-/-}$cells) and acentrosomal cells (RPEp53-/-SAS6 ${ }^{-/-}$and RPEp53/- $\mathrm{STIL}^{-/-}$cells) using the protein abundance quantification program Progenesis QI for proteomics. Variation in experimental conditions meant that the raw abundances (the sums of all unique normalized peptide ion abundances) of each protein on each run quantified by Progenesis QI for proteomics were normalized before subsequent analysis (Fig S1). On analysis of the normalized protein abundances of each FA protein between the control cells

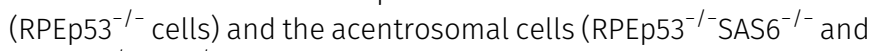
RPEp53 ${ }^{-/-} \mathrm{STIL}^{-/-}$cells) using the bioinformatics tool, the Ingenuity Pathway Analysis, we uncovered significant changes in the canonical pathways in FAs and that these are highly related to centrosome formation (Fig 4A). These findings indicate that centrosome formation dramatically affects the amount of various proteins present in FAs that are known to be involved in specific pathways; these are the actin cytoskeleton, integrin-based FA, and Rho-family small GTPases (Fig 4A). Finally, the normalized protein

Figure 2. The absence of the centrosome changes global microtubule dynamics in a migrating cell.

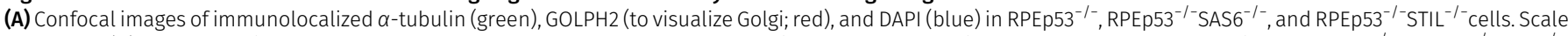

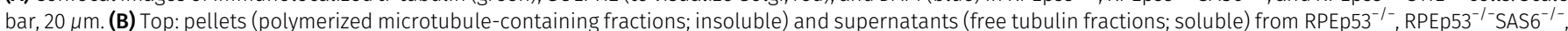
and RPEp53-/- STIL ${ }^{-/-}$cells were fractionated and then analyzed by Western blotting using antibodies against $\alpha$-tubulin ( $\alpha$-tub), acetylated tubulin (acetyl-tub), and

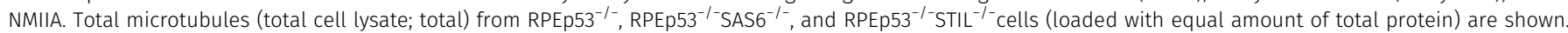
Bottom: (left) fold enrichment of $\alpha$-tubulin in the total cell lysate determined by Western blotting ( $n=5$ independent experiments); (right) fold of $\alpha$-tubulin in the polymerized microtubule-containing fractions (pellet; insoluble): free tubulin fractions (supernatant; soluble) as determined by Western blotting ( $\mathrm{n}=7$ independent experiments). Data are mean \pm SEM. ${ }^{*} P<0.05 ;{ }^{* *} P<0.001$; NS, no significance, all compared with RPEp53 ${ }^{-/-}$. (C) Confocal images of fluorescent EB3 comets obtained from

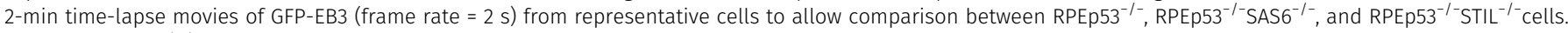
Scale bar, $20 \mu \mathrm{m}$. (D) Percentage of the population of microtubules whose dynamics were categorized into subpopulations based on the median values for microtubule growth speed and growth excursion lifetime in RPEp53 ${ }^{-/-}$cells. (E) Comparison of the average values for microtubule growth speed and growth excursion lifetime obtained

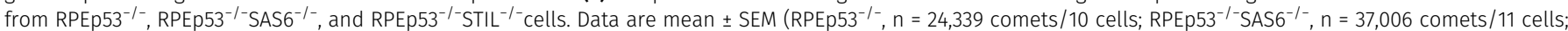
and RPEp53 ${ }^{-/-} \mathrm{STIL}^{-/-}, \mathrm{n}=43,213$ comets/14 cells). ${ }^{* *} \mathrm{P}<0.001$, all compared with RPEp53 ${ }^{-/-}$. (F) Confocal images of immunolocalized phalloidin (red), $\alpha$-tubulin (green),

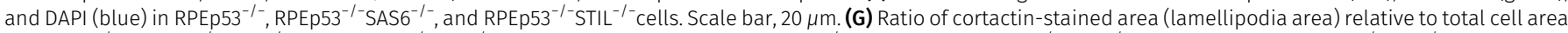

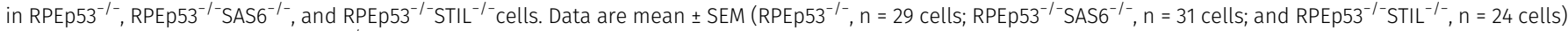
$* * * P<0.001$, compared with RPEp53/- 


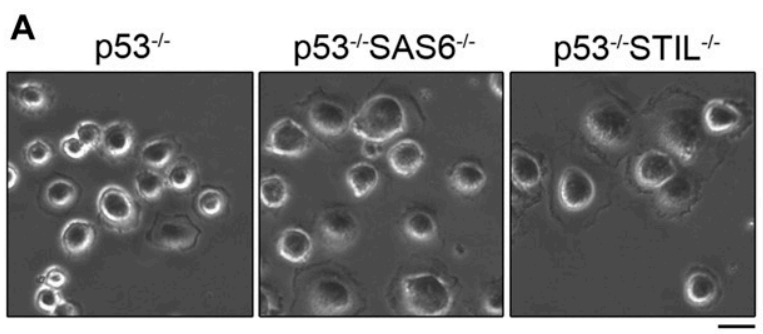

D
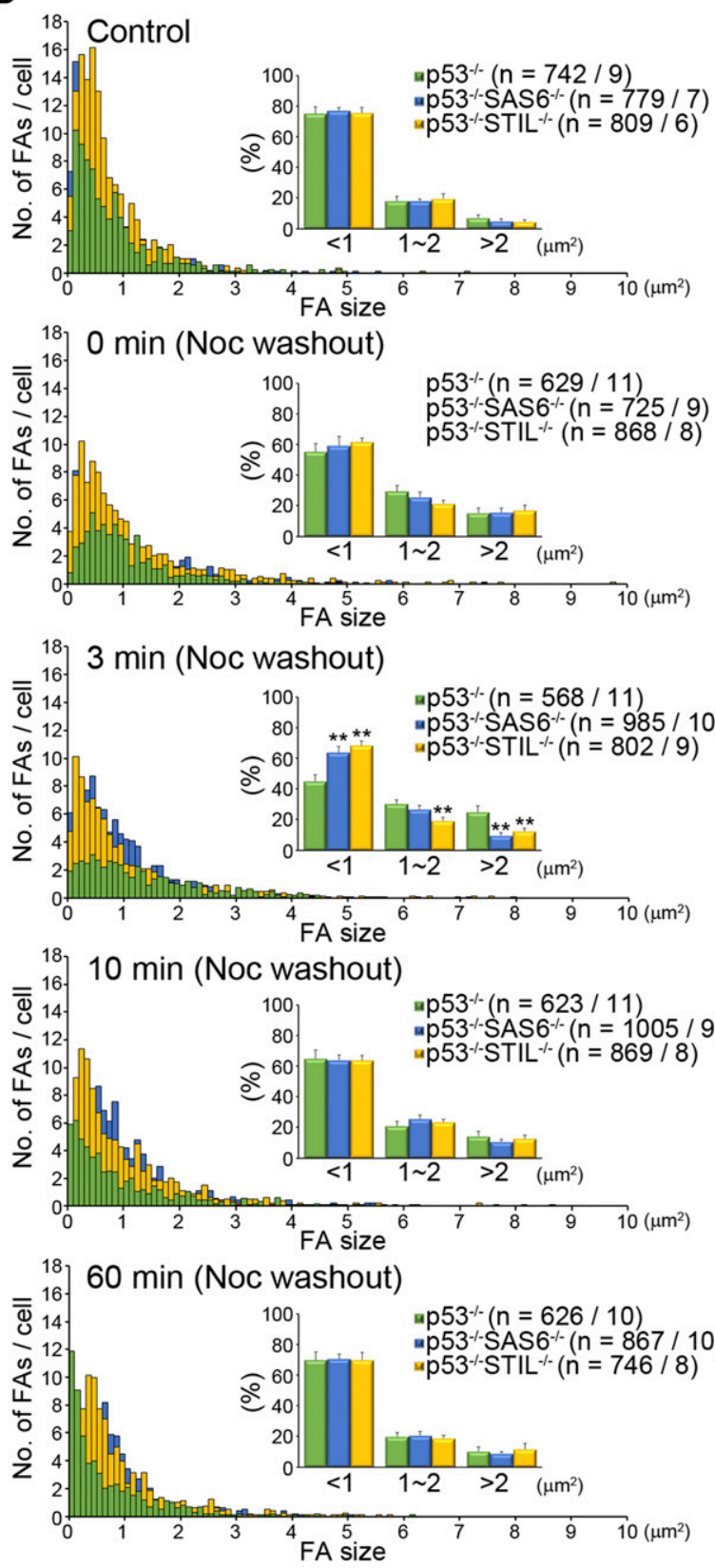

B
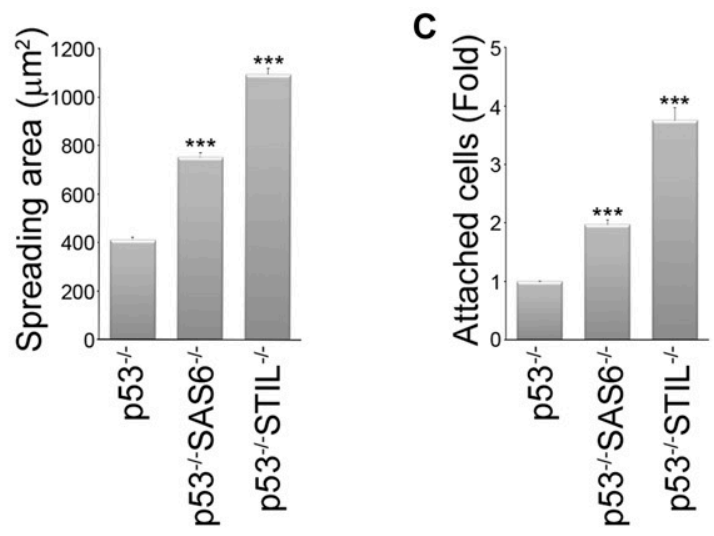

E
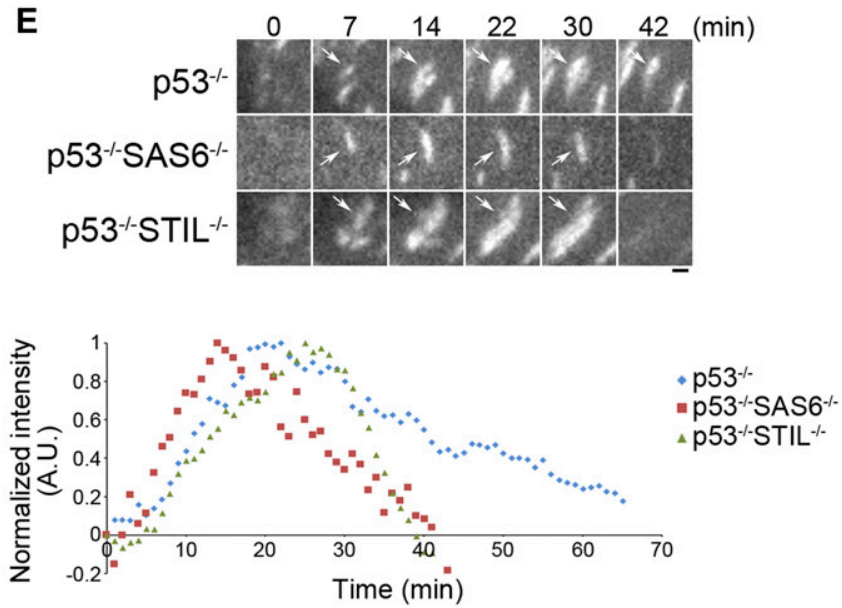

$\mathbf{F}$
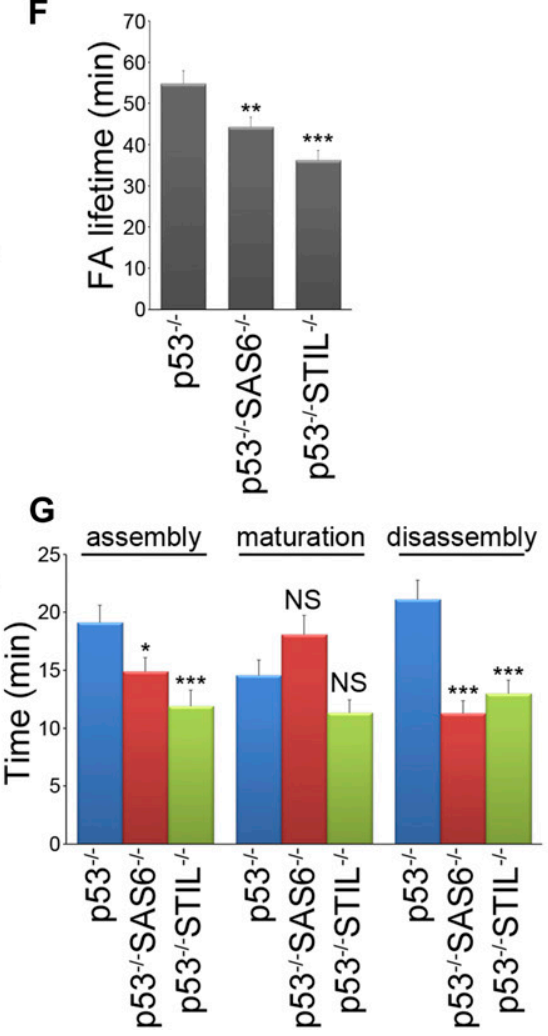

H

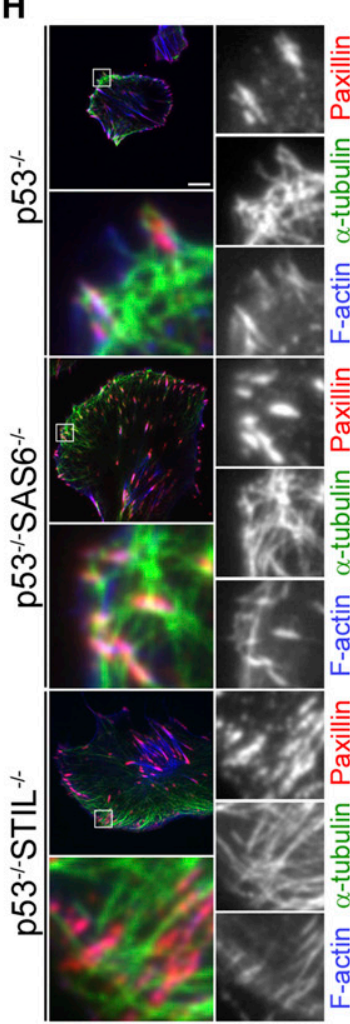


abundance of each protein on each run was calculated to determine the differences ( $P$-value; $t$ test) in abundance of each protein between the FAs of control cells and the acentrosomal cells (RPEp53-/-

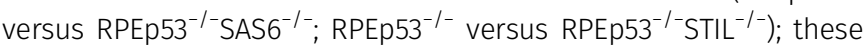
relative protein abundances were calculated as a ratio (the centrosome dependence ratio) to display the centrosome-modulated FA proteome (Tables S4 and S5). Of the proteins reproducibly identified in FAs of the control cells and the acentrosomal cells, those showing significant differences ( $P$-value $<0.05)$ in abundance between the

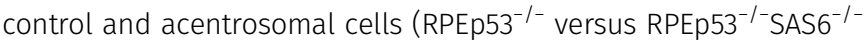
or RPEp53 $3^{-1-}$ versus RPEp53 ${ }^{-1-} \mathrm{STIL}^{-/-}$) were proteins that had ratio $>1$, indicating centrosome-dependent FA recruitment, and proteins that had ratio $<1$, indicating FA recruitment was inhibited by centrosome formation. The proteins without any significant difference ( $P$-value $\geq$ 0.05) are believed to represent minor centrosome-dependent changes in FA abundance and centrosome-independent FA recruitment. Thus, centrosome formation was shown to be able to influence FA enrichment of proteins, and this then leads to substantial changes in FA composition. This in turn modulates distinct pathways, namely, microtubule dynamics, integrin-based FA signaling, actin organization, and directed cell migration.

Directed cell migration in living cells is known to be regulated dynamically by Rho-family small GTPases. In the centrosomemodulated FA proteome (Tables S4 and S5), we found that proteins known to be involved in pathways related to Rac1-mediated actin polymerization and microtubule dynamics were strongly enriched in FAs of acentrosomal cells, compared with control cells (Fig 4B). Loss of the centrosome resulted in enrichment in FAs of Rac1, of Rac1 enhancers, of Rac1 effectors, and of various Rac1 downstream targets. The Rac1 enhancers that were recruited to the FAs of acentrosomal cells included Rac GEF TRIO (van Rijssel et al, 2012) $\left(\right.$ RPEp53 $3^{-/-}$versus RPEp53 ${ }^{-/-}$SAS6 $^{-/-}$: ratio $=0.6224$, -value $=$ 0.0097; RPEp53 ${ }^{-/-}$versus RPEp53 ${ }^{-/-} \mathrm{STIL}^{-1-}$ : ratio $=0.4299$, -value $=$ 0.0131 ) and Rac GEF modulator EPS8 (epidermal growth factor receptor kinase substrate 8) (Innocenti et al, 2002) (RPEp53-/- versus RPEp53 ${ }^{-1-}$ SAS6 $^{-1-}$ : ratio $=0.3979, P$-value $=0.0061 ;$ RPEp53 $^{-1-}$ versus RPEp53 ${ }^{-1-}$ STIL $^{-1-}$ : ratio $=0.0977$, P-value $\left.=0.0021\right)$. RhoA inhibitor RhoGDI (Rho GDP-dissociation inhibitor 1) (Del Pozo et al, 2002) $\left(\right.$ RPEp53 $3^{-/-}$versus RPEp53 ${ }^{-/-}$SAS6 $^{-/-}$: ratio $=0.3178$, $P$-value $=0.0224$; RPEp53 ${ }^{-/-}$versus RPEp53 ${ }^{-/-}$STIL $^{-1-}$ : ratio $=0.1336, P$-value $=0.0022$ ), which indirectly promotes Rac1 activation, was also found to be enriched in FAs when there was centrosome disruption. Furthermore, ARHGAP22 (Sanz-Moreno et al, 2008) (RPEp53-/- versus

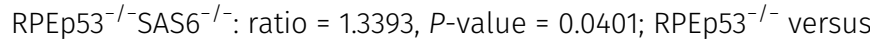
RPEp53 ${ }^{-/-} \mathrm{STIL}^{-1-}$ : ratio $=0.304$, -value $\left.=0.1944\right)$, which inhibits Rac1 activity, was found to be enriched in the FAs of control cells compared with acentrosomal cells. The Rac1 effectors found to be affected included IRSp53 (insulin receptor tyrosine kinase substrate p53) (Miki et al, 2000) (RPEp53 ${ }^{-/-}$versus RPEp53 ${ }^{-/-}$SAS6 $^{-1-}$ : ratio $=$ 0.0975, $P$-value $=0.0246 ;$ RPEp53 ${ }^{-/-}$versus RPEp53 $3^{-1-} \mathrm{STIL}^{-1-}$ : ratio $=$ 0.0217, P-value $=0.0154$ ), which serves as a mediator of Arp2/3dependent actin polymerization. The Rac1 downstream targets involved in actin treadmilling found to be affected included ARP2/3 complex (ARP2 [RPEp53 ${ }^{-/-}$versus RPEp53 ${ }^{-/-} \mathrm{SAS6}^{-/-}$: ratio $=0.6274$, $P$-value $=0.1191 ; \mathrm{RPEp53} 3^{-/-}$versus RPEp53 ${ }^{-/-} \mathrm{STIL}^{-1-}$ : ratio $=0.182$, $P$-value $=0.0173]$, ARP3 $\left[\right.$ RPEp53 ${ }^{-/-}$versus RPEp53 $3^{-/-} \mathrm{SAS6}^{-1-}$ : ratio $=$ 1.0736, $P$-value $=0.3131 ; \mathrm{RPEp53}^{-1-}$ versus RPEp53 ${ }^{-1-} \mathrm{STIL}^{-1-}$ : ratio $=$ 0.4301, $P$-value $=0.0021]$, ARPC2 [RPEp53 ${ }^{-/-}$versus RPEp53 ${ }^{-/-} \mathrm{SAS6}^{-1-}$ : ratio $=1.0516, P$-value $=0.4124 ; \mathrm{RPEp}^{-1-}$ versus RPEp53 ${ }^{-/-} \mathrm{STIL}^{-1-}$ :

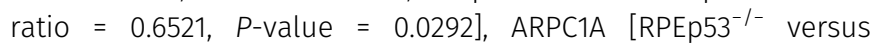
RPEp53 ${ }^{-1-}$ SAS6 $^{-1-}$ : ratio $=0.9594, P$-value $=0.4299 ;$ RPEp53 $^{-1-}$ versus RPEp53 ${ }^{-1-} \mathrm{STIL}^{-1-}$ : ratio $=0.3903, P$-value $\left.=0.00001\right], \mathrm{ARPC1B}$

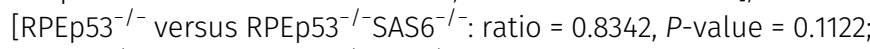
RPEp53 $^{-/-}$versus RPEp53 ${ }^{-/-} \mathrm{STIL}^{-/-}$: ratio $=0.7798$, -value $\left.=0.093\right]$, ARPC5L [RPEp53 $3^{-/-}$versus RPEp53 ${ }^{-/-}$SAS6 $6^{-1-}:$ ratio $=0.884, P$-value $=$ 0.2361; RPEp53 $3^{-/-}$versus RPEp53 ${ }^{-/-} \mathrm{STIL}^{-1-}$ : ratio $=0.4299$, -value $=$ 0.0091], ARPC3 [RPEp53 ${ }^{-/-}$versus RPEp53 ${ }^{-/-} \mathrm{SAS6}^{-/-}$: ratio $=1.1548$,

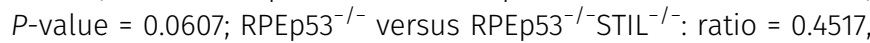
$P$-value $=0.1221]$, ARPC4 $\left[\right.$ RPEp53 $^{-/-}$versus RPEp53 ${ }^{-/-}$SAS6 $^{-1-}$ : ratio $=$ 1.1168, $P$-value $=0.2278 ;$ RPEp53 $3^{-/-}$versus RPEp53 ${ }^{-1-} \mathrm{STIL}^{-1-}:$ ratio $=$

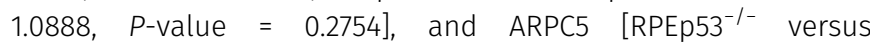

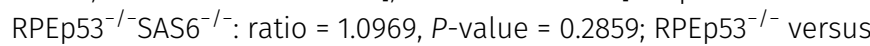
$\mathrm{RPEp53}^{-/-} \mathrm{STIL}^{-1-}$ : ratio $=0.6576, \quad$-value $\left.\left.=0.0434\right]\right)$, profilin

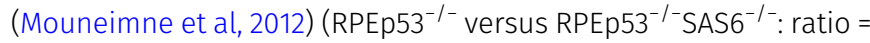
0.6461, $P$-value $=0.0183 ;$ RPEp53 ${ }^{-/-}$versus RPEp53 $3^{-/-} \mathrm{STIL}^{-1-}$ : ratio $=$ $0.2072, P$-value $=0.0002)$, cofilin (Oser \& Condeelis, 2009) $\left(\right.$ RPEp53 $3^{-1-}$ versus RPEp53 $3^{-1-} \mathrm{SAS6}^{-1-}$ : ratio $=0.6684, \mathrm{P}$-value $=0.068 ; \mathrm{RPEp53}^{-1-}$ versus RPEp53 ${ }^{-1-} \mathrm{STIL}^{-1-}$ : ratio $=0.2164, \mathrm{P}$-value $\left.=0.00001\right)$, and the actin monomer-binding protein CAP1 (Bertling et al, 2004) $\left(\right.$ RPEp53 $3^{-1-}$ versus RPEp53 $3^{-1-}$ SAS6 $6^{-1-}$ : ratio $=0.3477$, -value $=0.0067$; $\mathrm{RPEp53}^{-/-}$versus RPEp53 ${ }^{-/-} \mathrm{STIL}^{-/-}$: ratio $=0.113, \mathrm{P}$-value $=0.0076$ ) Immunoblotting of the FA fractions was able to validate the fact that there was negative regulation by centrosome assembly of Rac1, TRIO, GEF-H1, and integrin $\beta 1$ in FAs, whereas disruption of the centrosome's intact structure resulted in a significant increase in these proteins in the FA fractions, in both cases compared with the

Figure 3. The absence of the centrosome changes FA dynamics in a migrating cell.

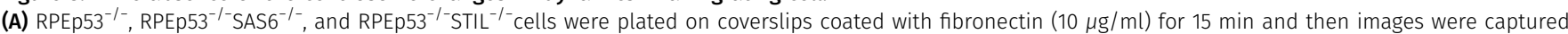
by phase-contrast microscopy. Scale bar, $20 \mu \mathrm{m}$. (B) The plot shows the area of cells spreading on coverslips coated with fibronectin (10 $\mu \mathrm{g} / \mathrm{ml}$ ) for $15 \mathrm{~min}$. Data are mean \pm

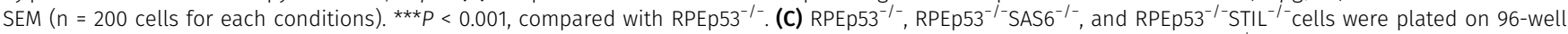
plates coated with fibronectin $(10 \mu \mathrm{g} / \mathrm{ml})$ for $30 \mathrm{~min}$ and then their cell attachment was measured. Fold of cells (relative to RPEp53 ${ }^{-/-}$cells) that remained attached to the fibronectin-coated plates. Data are mean \pm SEM ( $n=6$ independent experiments). ${ }^{* * *} P<0.001$, compared with RPEp53 ${ }^{-/-}$. (D) Size distribution of segmented FAS

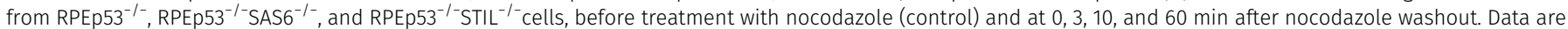
mean \pm SEM ( $n=$ number of FAs/number of cells). ${ }^{* *} P<0.01$, compared with RPEp53 ${ }^{-1-}$. (E) Top: time-lapse TIRF microscopy images of GFP-paxillin during FA turnover in

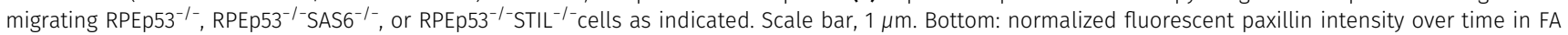

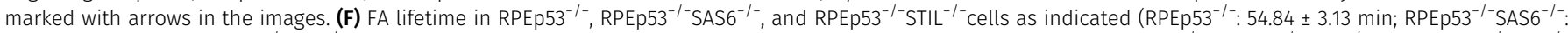

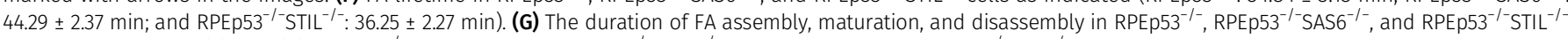

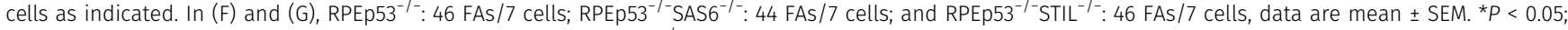
${ }^{* \star} P<0.01 ;{ }^{* \star} P<0.001$; NS, no significance, all compared with RPEp53-/- (H) TIRF microscopy images of immunolocalized $\alpha$-tubulin (green), paxillin (to visualize FAs; red),

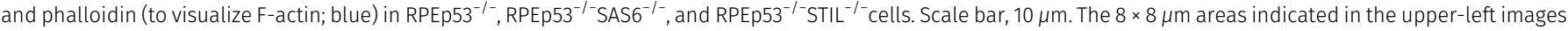
are magnified in the merged and right three images. 


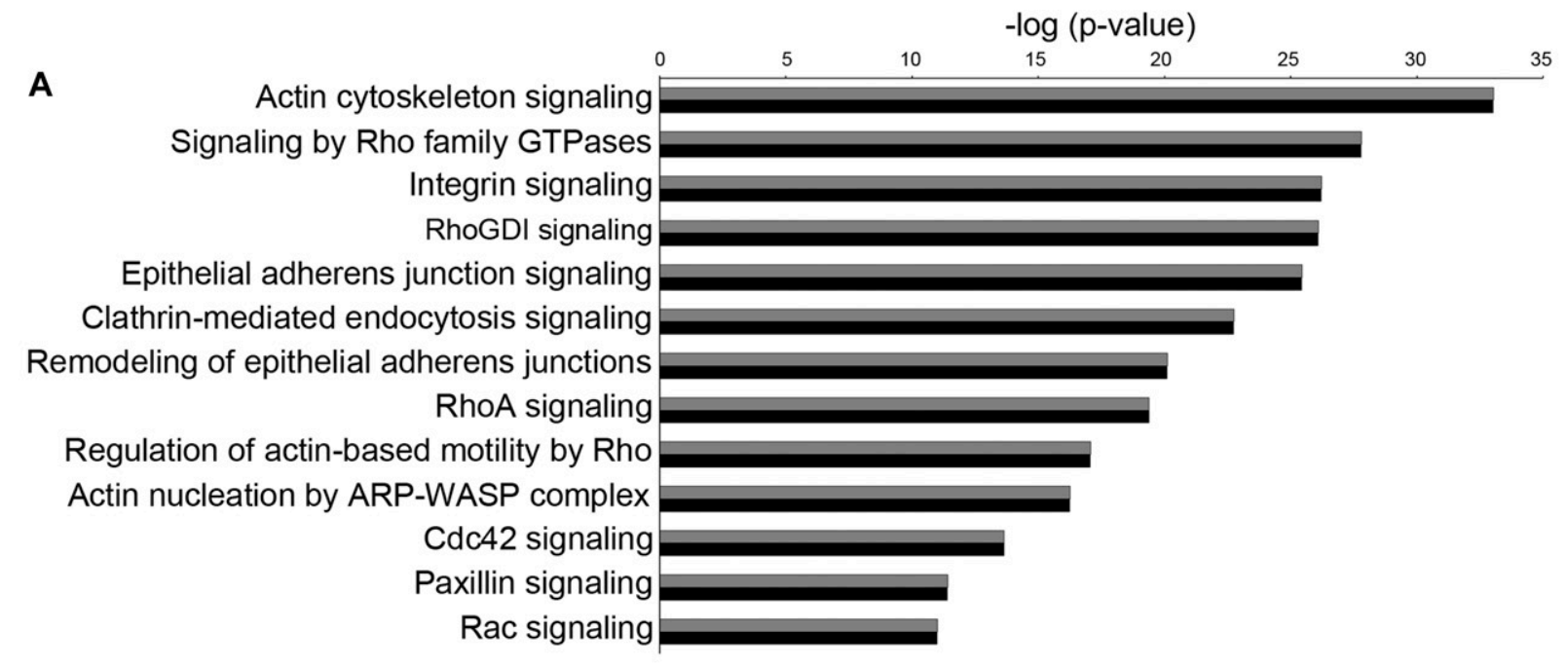

aFA proteins in RPEp53\% : FA proteins in RPEp53\%-SAS6 $\%$

- FA proteins in RPEp53\% : FA proteins in RPEp53\% ${ }^{-T_{I L}}{ }^{-/}$

B

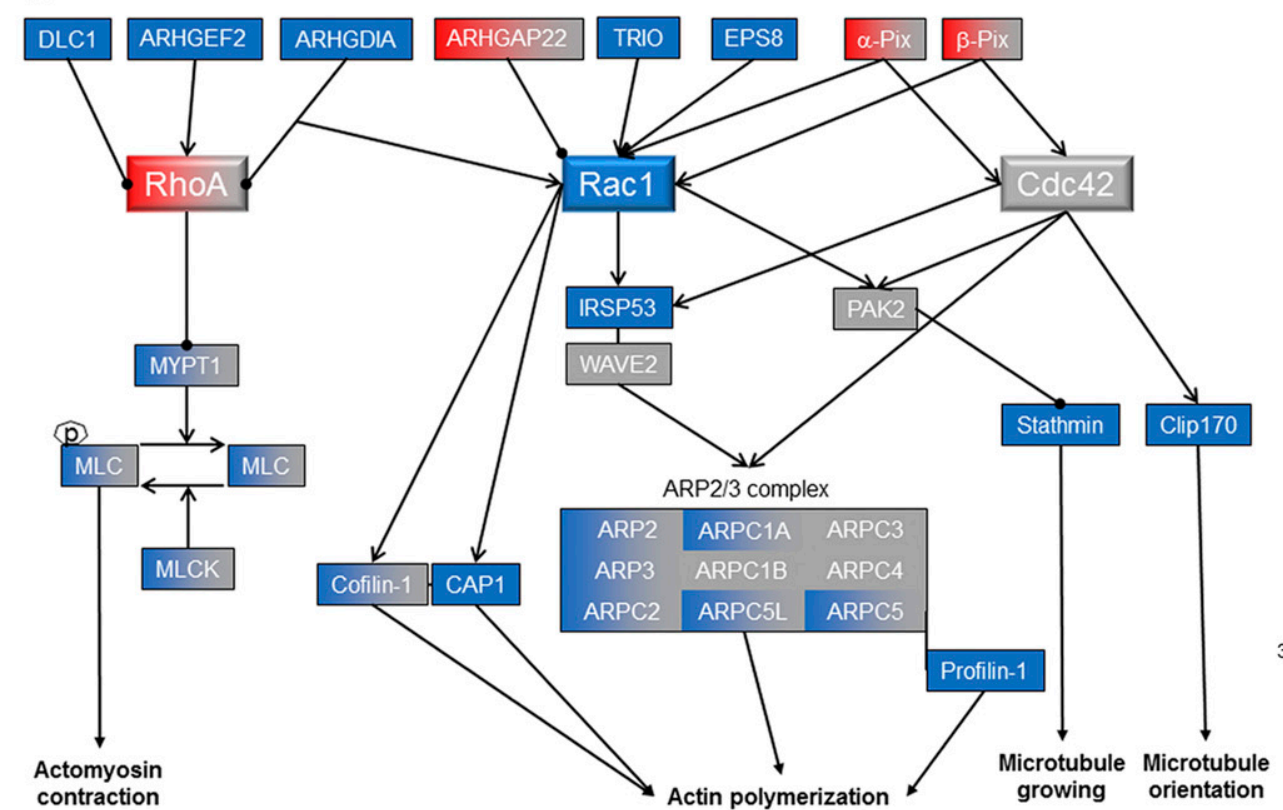

C

\section{$\underline{\text { Isolated FAs }}$}

这市 ल क

in ले लि

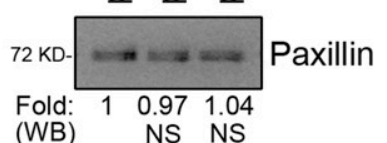

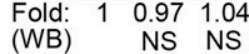

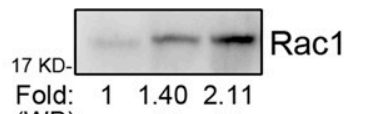

$\begin{array}{llll}\text { Fold: } & 1 & 1.40 & 2.11 \\ \text { (WB) } & & * * & * * *\end{array}$

Fold: $11.44 \quad 1.93$

(MS) $* * * *$

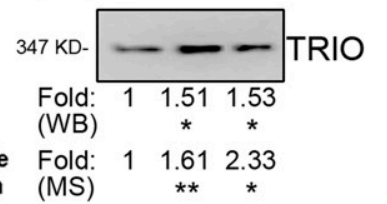

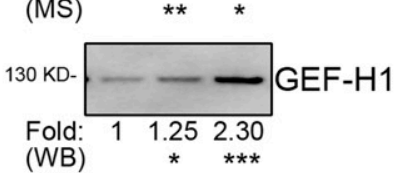

$\begin{array}{llll}\text { (WB) } & * & * & \\ \text { Fold: } & 1 & 1.68 & 4.24\end{array}$

(MS) * **

$130 \mathrm{KD}-\square-\longrightarrow \quad \begin{aligned} & \text { Integrin } \beta 1 \\ & \text { (active) }\end{aligned}$

Fold: 12.893 .68

(WB) $* * * * * *$

$130 \mathrm{KD}-\square$ Integrin $\beta 1$

Fold: $1 \quad 2.193 .01$

(WB) * **

Fold: 11.892 .73

(MS) ** ** 
control cells (RPEp53 $3^{-1-}$ ). These results are similar to those obtained by the proteome analysis (Fold [MS] $=1 /$ centrosome dependence ratio) (Fig 4C). Interestingly, active integrin $\beta 1$ accumulation in FAs was also found to be regulated by the centrosome based on the Western blotting of isolated FAs, which revealed an increased accumulation of active integrin $\beta 1$ in the FA fractions from acentrosomal cells (RPEp53 ${ }^{-/-} \mathrm{SAS6}^{-/-}$and $\mathrm{RPEp53}^{-/-} \mathrm{STIL}^{-1-}$ ) (Fig 4C) compared with control cells, supporting the results in Fig 3. These results were that centrosome disruption promotes FA formation, increase in the number of FAs, and increased cell adhesive ability. To further confirm if the centrosome modulates the downstream effect of Rac1, various cells were immunostained to detect $\gamma$-tubulin and F-actin, and the results showed that loss of the centrosome increased actin-based membrane protrusion (Fig 4D).

\section{Loss of the centrosome promotes excessive Rac1 activation and increased lamellipodial protrusion}

To better understand how the centrosome may negatively regulate Rac1 signaling and lamellipodial protrusion, we focused on the effect of centrosome disruption on the modulation of Rac1 activation via an exploration of this protein's role in the membrane protrusion/retraction cycle. Rac1-GTP pull-down assays were used to show that Rac1 activity was increased in acentrosomal cells (RPEp53 $3^{-/-} \mathrm{SAS6}^{-/-}$and RPEp53 ${ }^{-/-} \mathrm{STIL}^{-/-}$) compared with control cells (RPEp53-/-) (Fig 5A). Next, we investigated the spatial distribution of active Rac1. To do this, control and acentrosomal cells were transfected with the Rac1 FRET biosensor pTriEx4-Rac1-2G (Fritz et al, 2015) and then imaged to assess the Rac1 FRET/ECFP ratio. The results revealed that centrosome disruption resulted in extensive Rac1 activation across multiple regions, whereas active Rac1 was limited to the front of the control cells (Fig 5B). We next studied cell membrane dynamics in the control and acentrosomal cells. To do this, we performed live-cell imaging of fluorescently labeled CAAX (YFP-CAAX), which labels cell membrane. In the time-lapse image series obtained using YFP-CAAX, we tracked the dynamics of the cell boundary using a previously described computational platform CellGeo (Tsygankov et al, 2014) to assess changes in the cell boundary. These changes were colored to show areas of protrusion (white) versus areas of retraction (black) (Fig 5C). In the control cells, there was a front/back asymmetric distribution of persistent protrusion/retraction, which indicates polarized cell morphology. On the other hand, the membrane dynamics of the acentrosomal cells was random. Thus, the loss of the centrosome significantly enhanced Rac1 activation all over the cell, and this resulted in a large number of extensive lamellipodial protrusions, which suggests a possible link between Rac1 activation and microtubules in cells that have undergone centrosome disruption.

To investigate if microtubules contribute to the Rac1 activation induced by centrosome disruption, we carried out Rac1-GTP pulldown assays to examine Rac1 activity in the control and acentrosomal cells after treatment with nocodazole. The results revealed that Rac1 activity increased in acentrosomal cells, compared with control cells, and that the Rac1 activation induced by centrosome disruption was abolished by nocodazole treatment (Fig 5D). Thus, the presence of growing microtubules was a requirement for excessive Rac1 activation in acentrosomal cells, which implies that the increase in acentrosomal microtubules is delivering the specific signaling proteins that activate Rac1 excessively.

\section{The presence of TRIO on acentrosomal microtubules induces Rac1 activation and promotes lamellipodial protrusions}

To understand how growing microtubules are able to regulate Rac1 activity in a migrating acentrosomal cell, we focused on the GEFs (TIAM1, $\beta$-PIX, and TRIO), the GAPS (GIT1 and GIT2), and the GDIs (ARHGDIA and GDI2) within the Rho-family small GTPases. Examining Rac1 activity revealed that in cells expressing control shRNAs (non-silencing), Rac1 activity was increased by loss of the centrosome, whereas Rac1 activation induced by centrosome disruption was abolished in cells having undergone TRIO shRNA knockdown (Fig 6A), not in cells expressing TIAM1, $\beta$-PIX, GIT1, GIT2, ARHGDIA, or GDI2 knockdown shRNAs (Table S6). Immunolocalization of F-actin and the lamellipodia marker cortactin in above cells revealed that loss of the centrosome resulted in extensive enhancement of lamellipodial protrusions (Figs $2 \mathrm{G}$ and 6B) and that this was abrogated in cells expressing shRNAs that inhibited TRIO expression (Fig 6B). In addition, wound-healing migration assays revealed that silencing of TRIO reversed the defect in directional persistence induced by centrosome disruption (Fig 6C). Thus, TRIO is required for excessive Rac1 activation and the increase in lamellipodial formation, induced by centrosome disruption.

Dynamic microtubules control TRIO's GEF activity (van Haren et al, 2014), so we hypothesized that TRIO may preferentially be associating with acentrosomal microtubules to determine the subcellular localization of its GEF activity. To test this, we isolated

Figure 4. The absence of the centrosome modulates actin filaments organization via FA signaling in a migrating cell.

(A) The bar graph (scaled according to the ANOVA P-value) is representative of the results from the Ingenuity Pathway Analysis of the proteomics data from the various

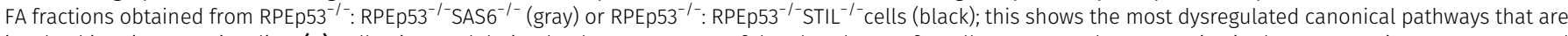
involved in migratory signaling. (B) Collective modulation by the centrosome of the abundance of small GTPases pathway proteins in the FAs. Proteins are represented by boxes that are color-coded according to their statistical significance categories and the magnitude of their ratio (as is indicated below each diagram). A P-value $<0.05$ ( $t$ test) is considered to indicate with high confidence a significant change. Of the proteins with $P$-value $<0.05$, the proteins with centrosome dependence ratio $>1$ were considered to undergo centrosome-dependent recruitment, whereas the proteins with centrosome dependence ratio $<1$ were considered to have their recruitment

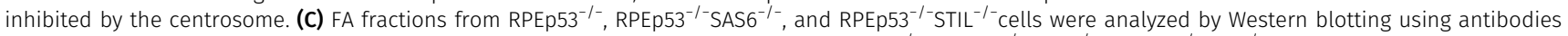
against paxillin, Rac1, TRIO, GEF-H1, active integrin $\beta 1$, and total integrin $\beta 1$. Fold (WB) (RPEp53 ${ }^{-1-}$ : RPEp53 ${ }^{-1-}$ SAS6 $^{-1-}$ : RPEp53 ${ }^{-1-}$ STIL ${ }^{-1}$ cells) indicates the fold enrichment of paxillin (1: $0.97 \pm 0.12: 1.04 \pm 0.18 ; n=12$ independent experiments), Rac1 (1: 1.40 $\pm 0.13: 2.11 \pm 0.25 ; n=22$ independent experiments), TRIO (1: 1.51 $\pm 0.24: 1.53 \pm 0.25 ; n=10$ independent experiments), GEF-H1 (1:1.25 $\pm 0.10: 2.30 \pm 0.29 ; n=16$ independent experiments), active integrin $\beta 1(1: 2.89 \pm 0.44: 3.68 \pm 0.40 ; n=11$ independent experiments), and total integrin $\beta 1$ (1: 2.11 $\pm 0.47: 2.82 \pm 0.50 ; n=9$ independent experiments) in isolated FA fractions as determined by Western blotting (loaded with equal amount of total protein, data are mean \pm SEM). Fold (MS) indicates fold enrichment of the indicated proteins in the various isolated FA fractions as determined by LC-MS/MS.

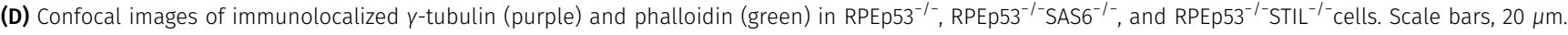




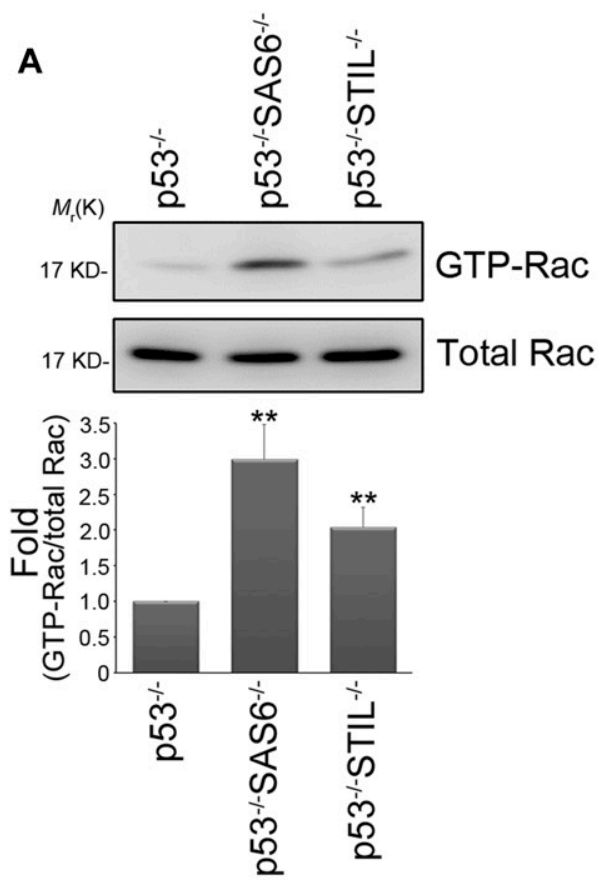

B
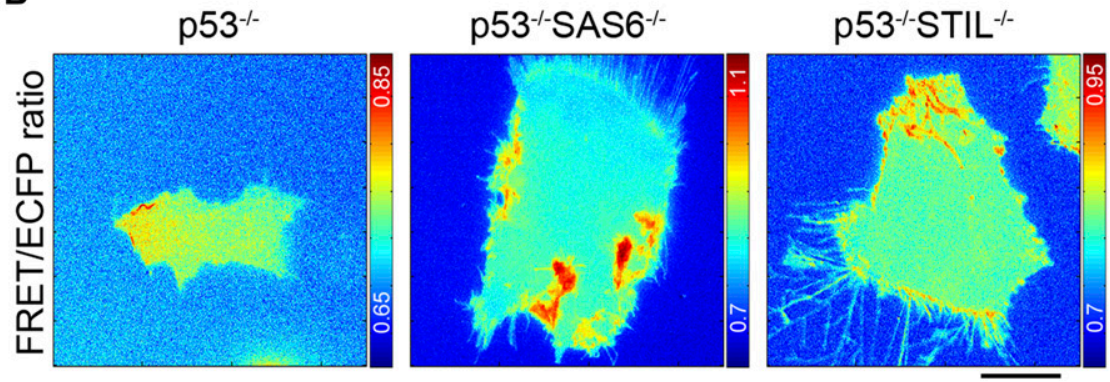

C
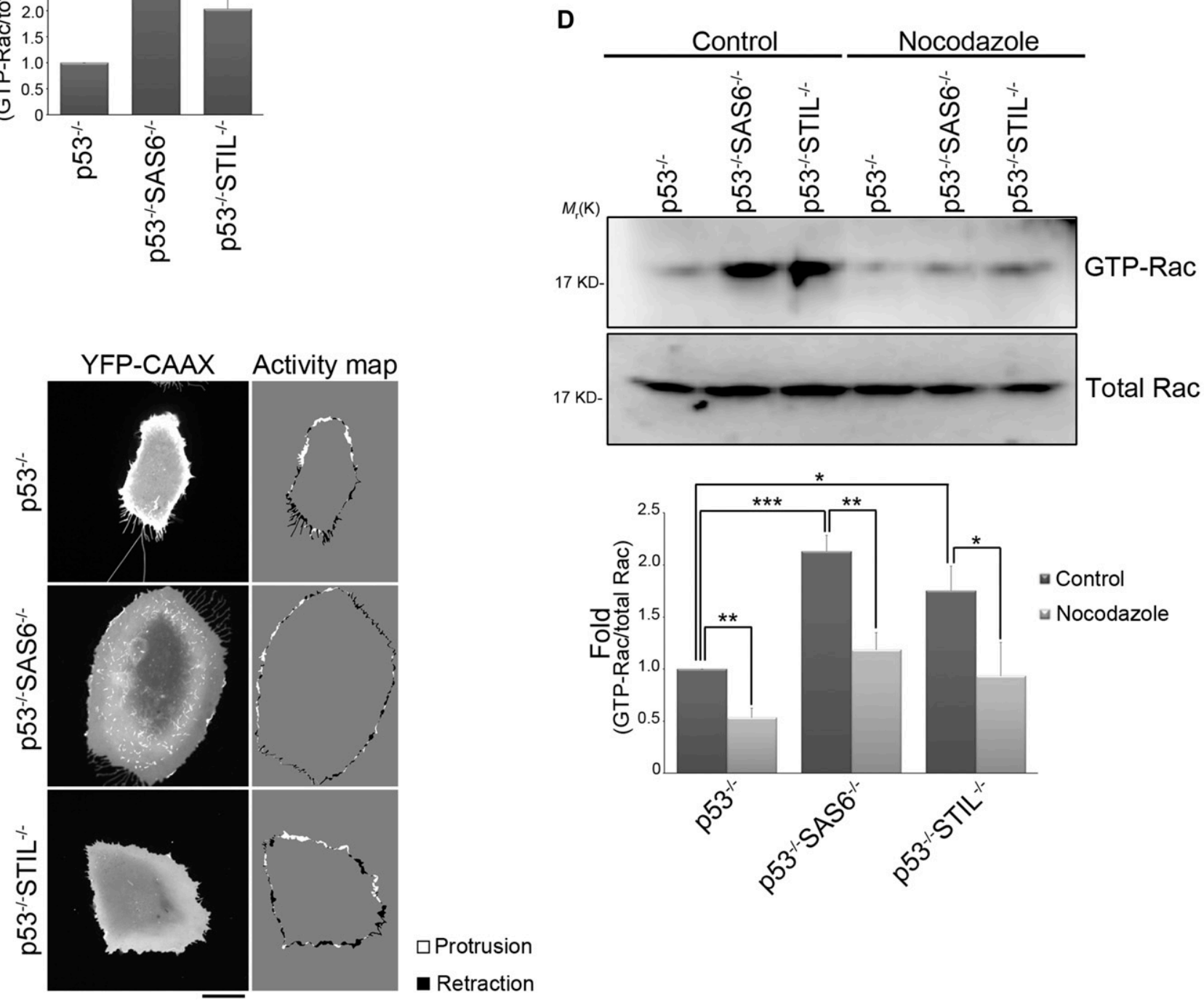

Figure 5. The absence of the centrosome promotes small GTPase Rac1 activation via acentrosomal microtubules.

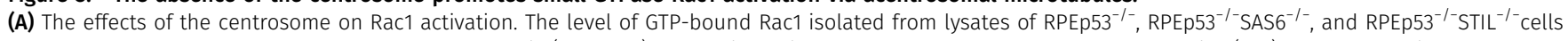
by GST-PAK-CRIB pull-down and the amount Rac1 protein (total Rac) present in the input lysate were detected by Western blotting (top). Fold changes in the level of GTPRac relative to total Rac determined by Western blotting is indicated below the blots. Data are mean $\pm \mathrm{SEM}$ ( $\mathrm{n}=8$ independent experiments). ${ }^{* *} P<0.01$, compared

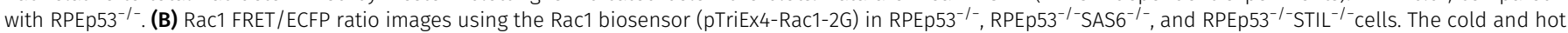
colors in the color bar represent low and high Rac1 activity levels, respectively. Bar, $20 \mu \mathrm{m}$. (C) Images of YFP-CAAX and a cell protrusion/retraction (white/black,

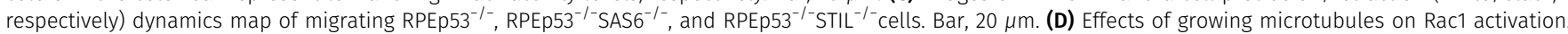

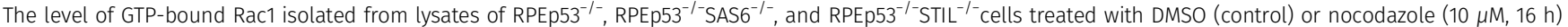
by GST-PAK-CRIB pull-down and the protein level of Rac1 (Total Rac) in the input lysate were detected by Western blotting (top). Fold changes in the levels of GTP-Rac relative to total Rac determined by Western blotting is indicated below the blots. Data are mean \pm SEM $\left(n=5\right.$ independent experiments). ${ }^{*}<0.05 ;{ }^{* \star} P<0.01 ;{ }^{* \star *} P<0.001$. 
Life Science Alliance

A
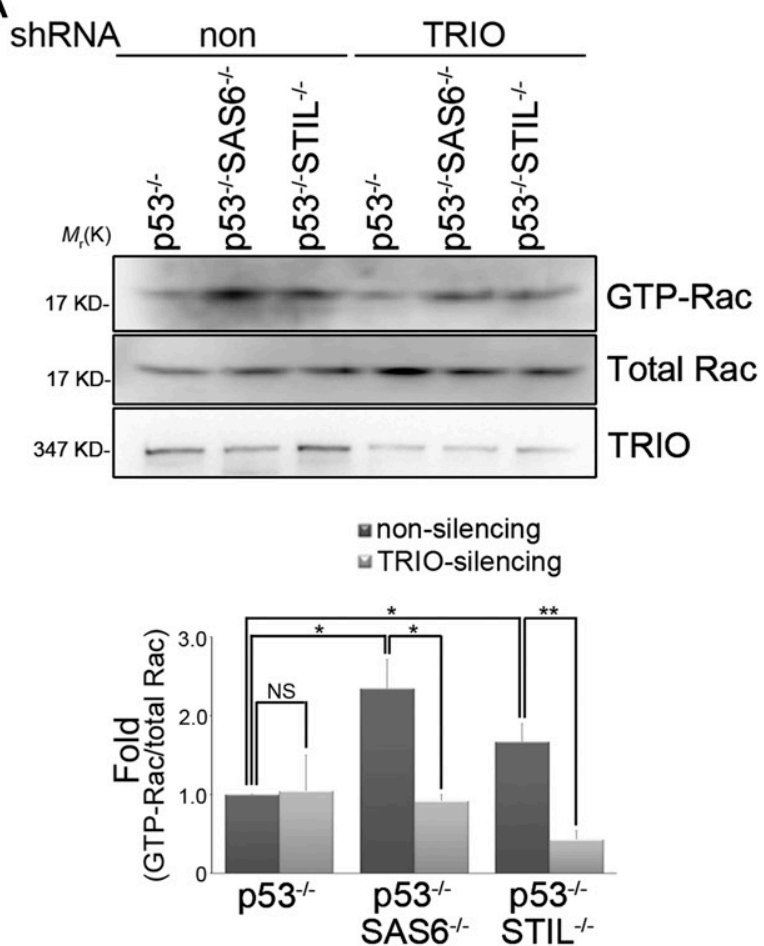

C

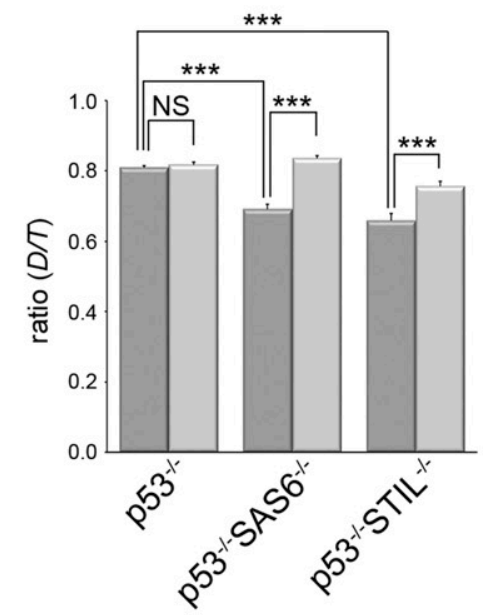

D

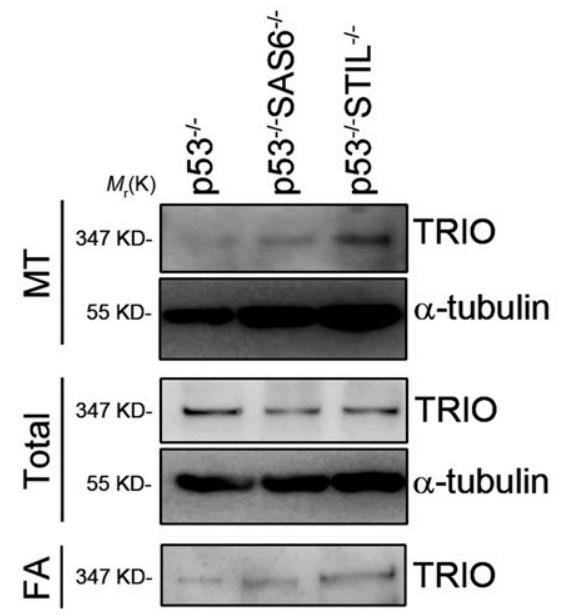

B

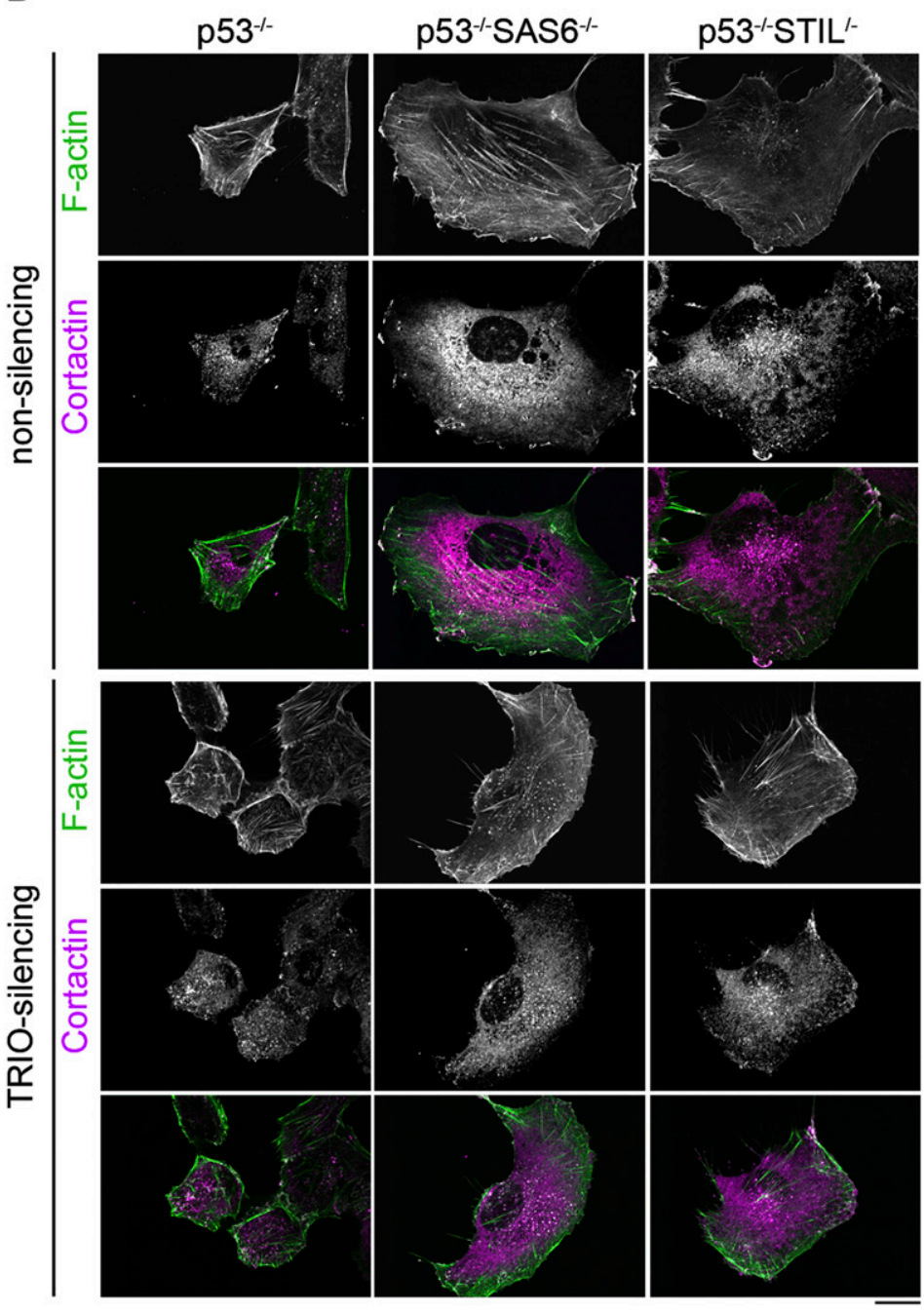

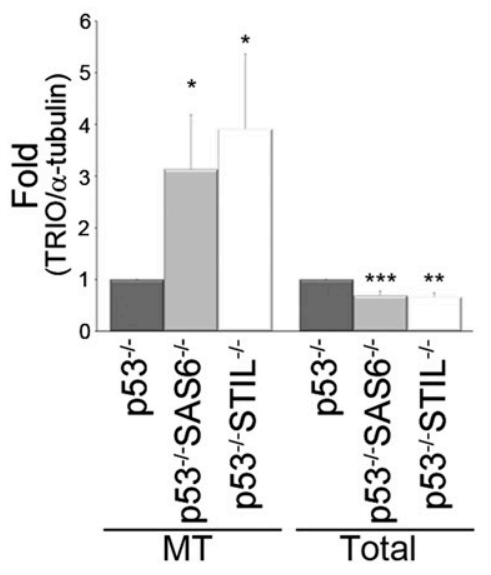

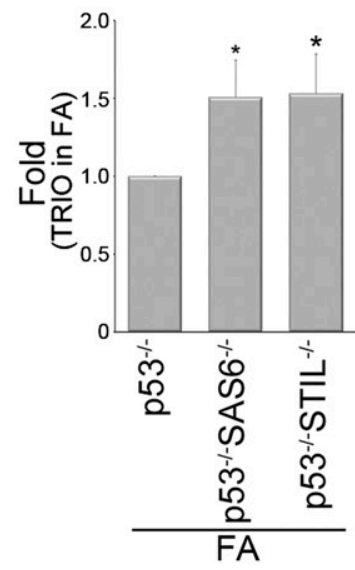


microtubule fractions from control (RPEp53-/- cells) and acen-

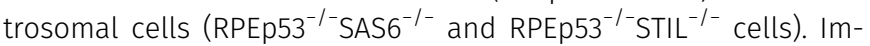
munoblotting of these microtubule fractions showed that there was increased TRIO accumulation in polymerized microtubules when there was centrosome disruption. Specifically, the loss of the centrosome resulted in a 3.1-fold increase in the presence of TRIO in the polymerized microtubules of RPEp53 ${ }^{-1-} \mathrm{SAS}^{-1-}$ cells and a 3.9-fold increase in the presence of TRIO in the polymerized microtubules of RPEp53-/- $\mathrm{STIL}^{-/-}$cells, both compared with control cells (RPEp53-/- cells) (Fig 6D). Next, we isolated the various FA fractions to confirm the results shown in Fig $4 \mathrm{~B}$ and $\mathrm{C}$, namely, that TRIO was enriched in the FAs from acentrosomal cells (Fig 6D). It was hypothesized by us that TRIO is delivered to FAs via the acentrosomal microtubules. To test this, we examined the FA localization of a TRIO mutant (TRIO-SRNN), which mutates the first SXIP motif (SRIP to SRNN) to abolish the binding of full-length TRIO to the microtubule plus end (van Haren et al, 2014). Analysis of mApple-paxillin and GFP-TRIO versus GFP-TRIO-SRNN revealed that GFP-TRIO, but not GFP-TRIO-SRNN, was localized in the paxillinmarked FAs of acentrosomal cells (RPEp53-/-SAS6 ${ }^{-1-}$ cells) (Fig 7A). These findings indicate that the delivery of TRIO to FAs depends on its interaction with the plus ends of acentrosomal microtubules. To further determine whether acentrosomal microtubule-delivered TRIO is required for its GEF activity, we altered TRIO expression and analyzed the effect of this on directed cell migration. Woundhealing migration assays revealed that in cells expressing the control shRNA, the directional persistence of migrating cells was suppressed by centrosome disruption (Figs 1D, 6C, and 7B). On the other hand, these polarity defects were rescued by TRIO knockdown (Figs 6C and 7B). This recovery was suppressed by reexpressing GFPTRIO, but not reexpression of GFP-TRIO-SRNN (Fig 7B). Taken together, these findings indicate that dynamic acentrosomal microtubules are able to deliver TRIO to the targeted FAs and this positively regulates TRIO's GEF activity, which in turn increases the activation of Rac1, enhances lamellipodia formation, and negatively regulates cell polarity during directed cell migration.

\section{Discussion}

Our study profiles centrosome-dependent FA composition changes and has uncovered a new role for the centrosome in regulating balance within the microtubule system that allows correct FA signaling and the maintenance of directed cell migration. To understand the role of the centrosome in FA-mediated signaling, we profiled the centrosome-dependent FA proteome, and this revealed that the centrosome collectively modulates the abundance of various functional modules of proteins (Fig 4). We then focused on small GTPase Rho signaling and showed that the loss of the centrosome increases the abundance of proteins involved in the Rac1 regulatory module of the FAs, including the protein Rac1 GEF TRIO. Loss of the centrosome was found to promote the formation of acentrosomal microtubules (Fig 2B), which are involved in delivering TRIO to FAs (Fig 7A). In acentrosomal cells, microtubule-delivered TRIO governs specific aspects of FA signaling, enhances Rac1 activation, and disrupts directed cell migration. In the present study, we have demonstrated for the first time that the presence of a centrosome restricts the assembly of acentrosomal microtubules by forming centrosomal microtubules, which in turn leads to correct activation of Rac1 and appropriate directed cell migration (Fig 7C).

The discovery of the centrosome regulates cell polarization in a migrating cell via acentrosomal microtubule-controlled FA signaling is surprising. In spite of the fact that the centrosome has been known for a considerable time to control cell polarization and motility, it has been generally thought that the centrosome releases signals that affect polarization. Experiments using laser microirradiation in 1984 provides the first evidence for the importance of the centrioles in directed cell migration (Koonce et al, 1984). Several studies using advanced techniques to carry out centrosome ablation have also confirmed that one of the centrosome's functions is the maintenance of a polarized microtubule network that allows cell migration in a specific direction (Wakida et al, 2010; Zhang \& Wang, 2017). Furthermore, recent evidence has suggested that the centrosome modulates motile cell polarization via emerging acentrosomal microtubules (Vinogradova et al, 2012). Our findings support this notion because we have found that centrosome loss promotes the assembly of acentrosomal microtubules that then attach to FAs; this up-regulates a Rac1 regulatory module in FAs and significantly enhances Rac1 activity. The result is that the cell loses its ability to maintain polarization during directed cell migration. Thus, our results provide the missing link that explains how the centrosome controls the coupling between Rac1 and actin polymerization at the cell front via a positive feedback loop to ensure cell polarity stability; specifically, this is done by restricting the assembly of acentrosomal microtubules. However, whether other mechanisms related to FA-mediated signaling are able to alter cell polarization or whether this is related to Cdc42 activity in acentrosomal cells has not yet been determined.

\section{Figure 6. The expression of TRIO is required for centrosome-modulated Rac1 activation.}

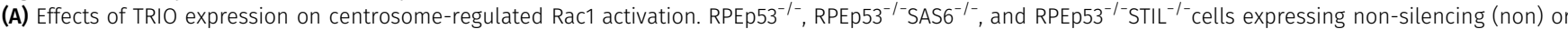
TRIO-silencing (TRIO) shRNAs were analyzed. The level of GTP-bound Rac1 isolated from lysates by GST-PAK-CRIB pull-down, the protein level of Rac1 (total Rac), and the amount of TRIO in the input lysate were detected by Western blotting (top). The fold changes in levels of GTP-Rac relative to total Rac determined by Western blotting are indicated below the blots. Data are mean $\pm \operatorname{SEM}\left(n=4\right.$ independent experiments). ${ }^{*} P<0.05$; ${ }^{* *} P<0.01$; NS, no significance. (B) Confocal images of

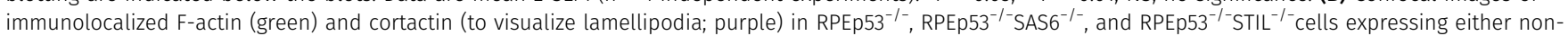

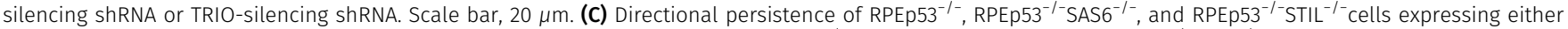

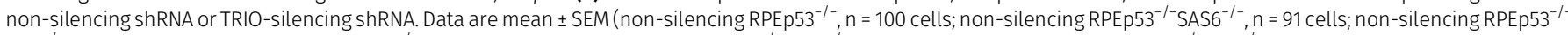

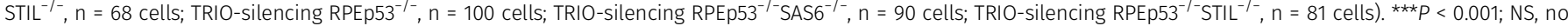
significance. (D) (Left) Western blot analysis of the microtubule fractions (polymerized microtubule-containing fraction; MT), the total cell lysates (total), and the FA

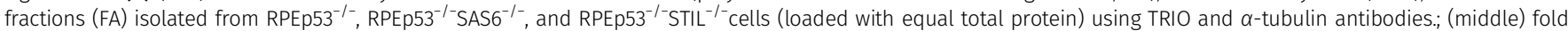
changes in the amount of TRIO: $\alpha$-tubulin in the isolated microtubule-containing fractions (MT) and in total cell lysates (total) were determined by Western blotting; (right) fold changes of TRIO in the FA fractions (FA) determined by Western blotting. Data are mean \pm SEM (microtubule isolation, $n=17$ independent experiments; total cell

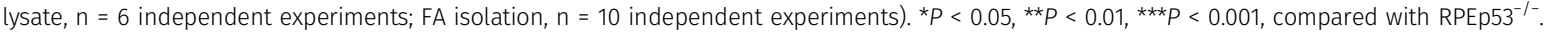


A

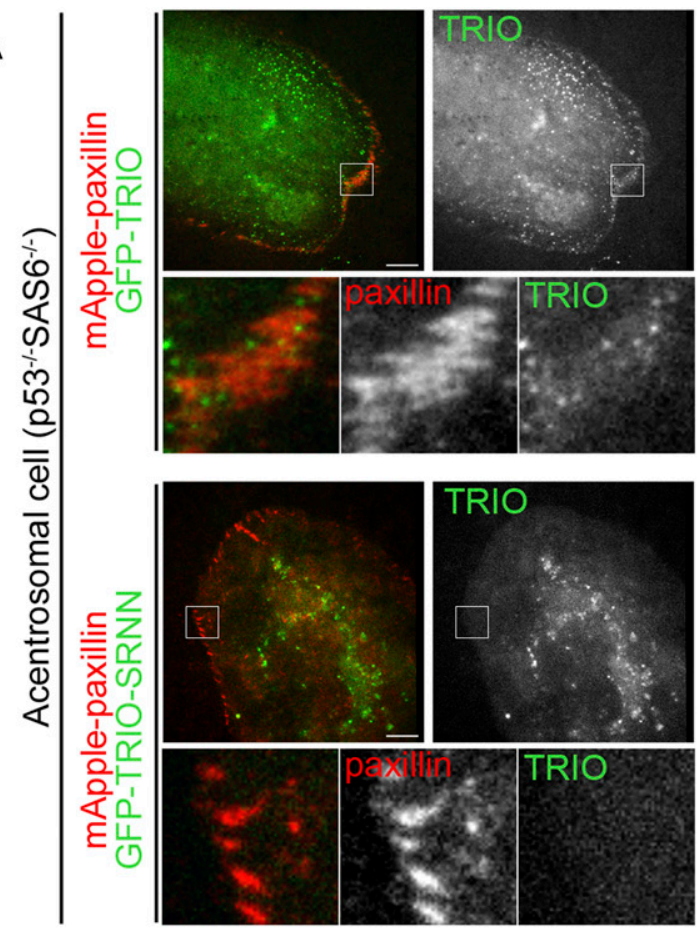

C
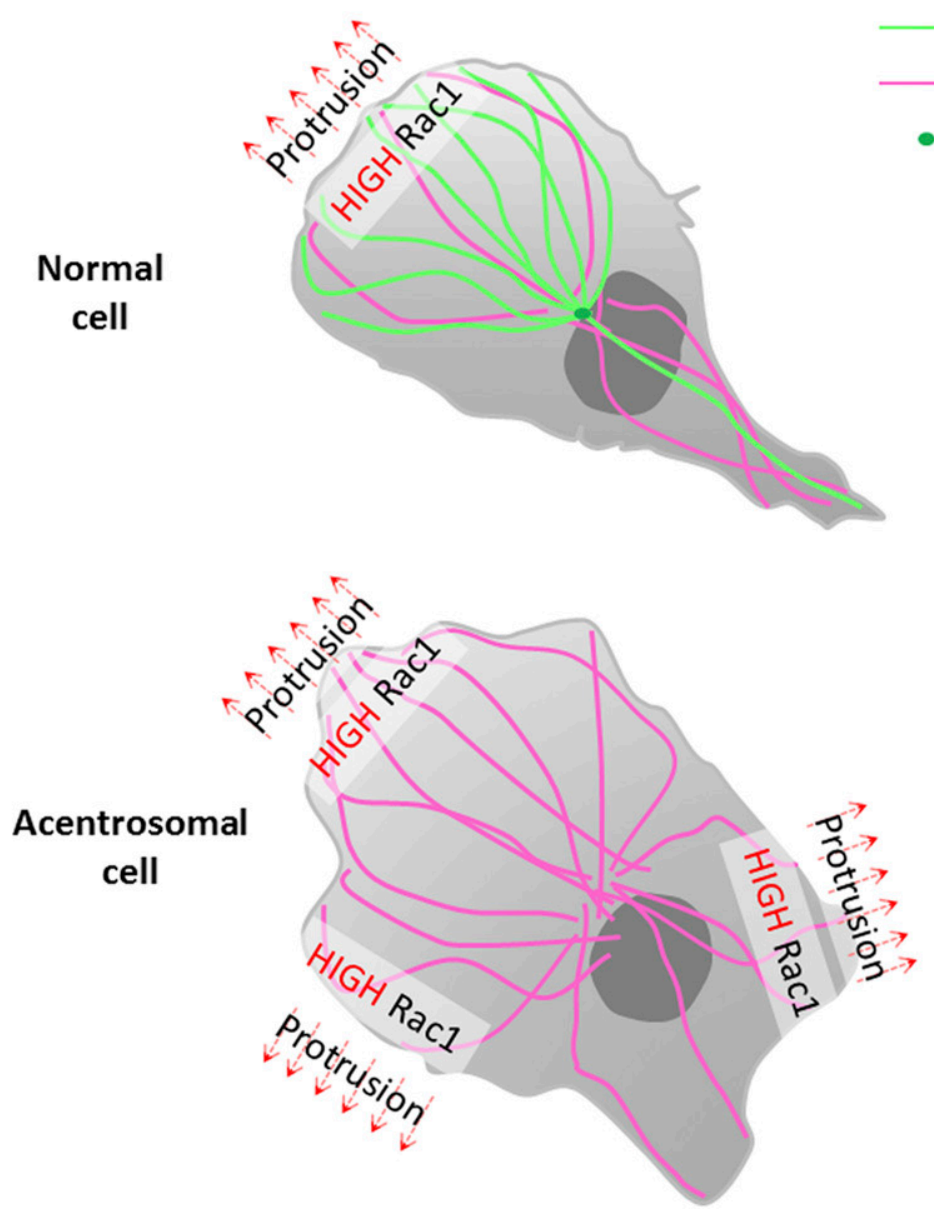

B

a non-silencing

- TRIO-silencing

a TRIO-silencing + GFP-TRIO

u TRIO-silencing + GFP-TRIO_SRNN

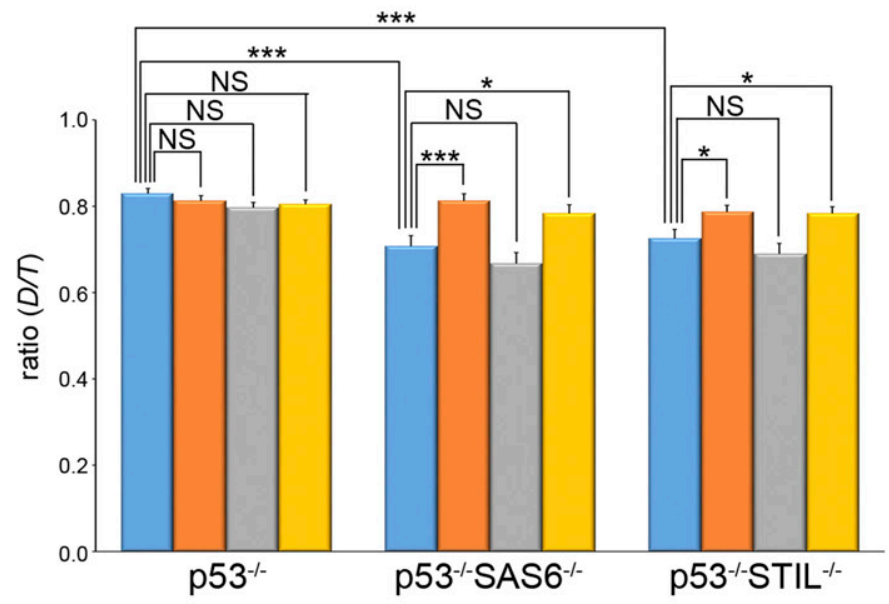

Centrosomal microtubules
Acentrosomal microtubules

- Centrosome

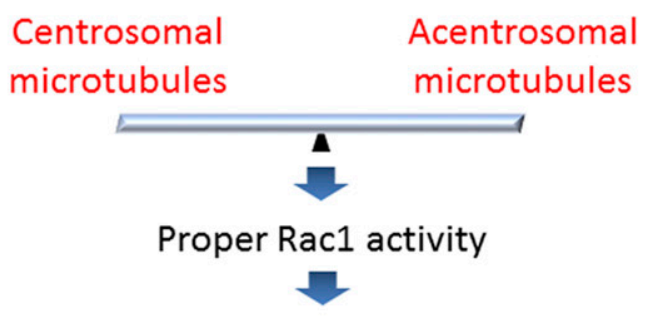

Directed cell migration

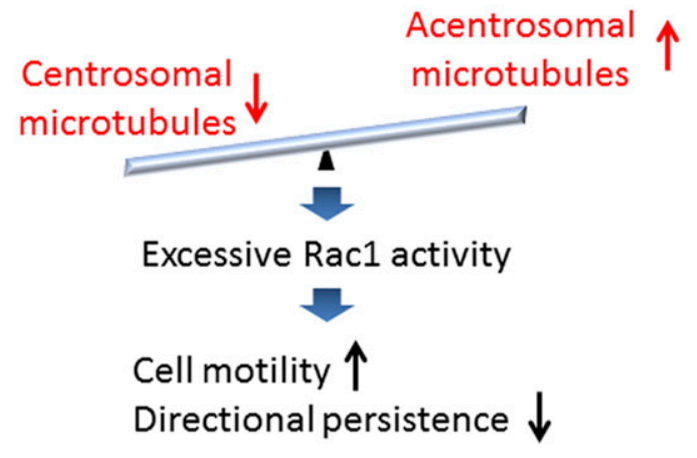


Our study has also revealed a previously unrecognized role for the Rac GEF TRIO protein; our findings place it in a physiologically relevant context related to centrosome-controlled cell polarity during directed cell migration. Compared with the control cells, TRIO shows a preference for associating with microtubules and FAs in acentrosomal cells (Fig 6D), which supports the notion that TRIO is likely to be a microtubule-associated protein involved in Rac1 activation (van Haren et al, 2014) and a component in FAs (Zaidel-Bar \& Geiger, 2010; Zaidel-Bar et al, 2007) via its association with filamin A (Bellanger et al, 2000), FAK (Medley et al, 2003), LAR (Debant et al, 1996), Rac1 (Bateman et al, 2000; Bellanger et al, 1998; Debant et al, 1996; Gao et al, 2001; Newsome et al, 2000), and RhoA (Bellanger et al, 1998; Medley et al, 2000). We have confirmed that TRIO is delivered to FAs via its interaction with acentrosomal microtubules plus ends (Fig 7A), but we still do not know how TRIO is able to choose to target microtubules and FAs in acentrosomal cells. A recent study has revealed that in the absence of centrosome, a specific microtubule minus end-binding protein CAMSAP2 (calmodulin-regulated spectrinassociated protein) is involved in organizing and stabilizing microtubule minus ends to maintain the density and mass of acentrosomal microtubules (Wu et al, 2016). In addition, we were able to detect more acetylated tubulins within the polymerized microtubules of centrosome-depleted cells (Fig 2B). These findings indicate that acentrosomal microtubules most likely have specific posttranslational modifications and/or are able to associate with a number of specific proteins. Because posttranslational modifications that act through microtubule-associated proteins or motors are able to directly tune the stability and dynamics of microtubules (Portran et al, 2017), this may be why acentrosomal microtubules behave differently in terms of microtubule stability and dynamics compared with centrosomal microtubules. In addition, it is possible that the TRIO protein chooses to associate with microtubules and FAs in acentrosomal cells by recognizing specific associated proteins and/or by targeting the posttranslational modifications of microtubules and FAs in acentrosomal cells.

\section{Materials and Methods}

\section{Cells}

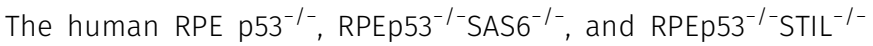
cells were kindly provided by Dr. Won-Jing Wang (National Yang-

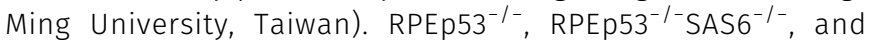

RPEp53 $3^{-1-} \mathrm{STIL}^{-/-}$cells stably expressing non-silencing, TRIO, GIT1, GIT2, TIAM1, $\beta$-PIX, ARHGDIA, or GDI2 shRNA were generated using a lentiviral shRNA system according to the manufacturer's instructions (National RNAi Core Facility Platform/Academia Sinica; Open Biosystems/Thermo Fisher Scientific). A Nucleofector Kit V (Lonza) was used for transient transfections. For all experiments, the cells were seeded on $10 \mu \mathrm{g} / \mathrm{ml}$ fibronectin-coated coverslips or plates.

\section{Plasmids}

Expression silencing of the following proteins was achieved using the following constructs: TRIO (TRCN0000196250; National RNAi Core Facility PlateForm), GIT1 (RHS4430-98911843; Open Biosystems), GIT2 (RHS4430-98476273; Open Biosystems), TIAM1 (RHS4430-98842140; Open Biosystems), $\beta$-PIX (RHS4430-98911853; Open Biosystems), ARHGDIA (TRCN0000008003; National RNAi Core Facility Platform), and GDI2 (RHS4430-99148519; Open Biosystems). The Rac1 biosensor (pTriEx4-Rac1-2G) was purchased from Addgene (\#66110).

\section{Antibodies}

Mouse anti-SAS6, rabbit anti-cortactin, and mouse anti- $y$-tubulin: Santa Cruz; rabbit anti-STIL and mouse anti-TRIO: Abcam; rabbit antiGEF-H1 and rabbit anti-integrin $\beta 1$ (total form), mouse anti-GAPDH, rabbit anti-GOLPH2, rabbit anti- $\beta$-actin, rabbit anti-paxillin, mouse anti- $\gamma$-tubulin, and rabbit anti-NMIIA: GeneTex; mouse anti- $\alpha$-tubulin and mouse anti-acetylated tubulin: Sigma-Aldrich; rat anti- $\alpha$-tubulin: ABD; mouse anti-paxillin: BD; mouse anti-Rac1 and mouse anti-integrin $\beta 1$ (active form): Millipore; rabbit anti-TRIO, Alexa Fluor 488 phalloidin, Alexa Fluor 488-anti-rat IgG, Alexa Fluor 488-anti-rabbit IgG, Alexa Fluor 488-anti-mouse IgG, Alexa Fluor 568-anti-mouse IgG, Alexa Fluor 568anti-rabbit IgG, Alexa Fluor 680-anti-rabbit IgG, and DAPI: Thermo Fisher; and HRP-AffiniPure mouse anti-rabbit IgG and HRP-AffiniPure goat anti-mouse IgG: Jackson ImmunoResearch.

\section{Reagents}

Nocodazole (Sigma-Aldrich).

\section{FA isolation}

The protocol for FA isolation was carried out as described previously (Kuo et al, 2011, 2012). Briefly, RPE cells were plated on

\section{Figure 7. Effects of TRIO on cell migration.}

(A) TIRF microscopy images of RPEp53 $3^{-1-} \mathrm{SAS}^{-1-}$ cells expressing mApple-paxillin (red) with either GFP-TRIO (green) or GFP-TRIO-SRNN (green). Scale bar, $10 \mu \mathrm{m}$. The

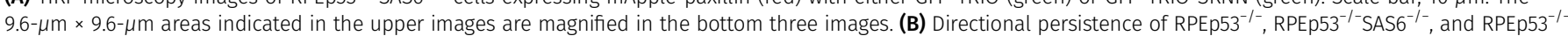
$\mathrm{STIL}^{-/-}$cells expressing non-silencing shRNA or TRIO-silencing shRNA alone or together with GFP-TRIO or GFP-TRIO-SRNN. Data are mean \pm SEM (non-silencing RPEp53-/- $\mathrm{n}=57$

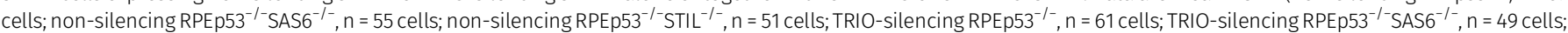

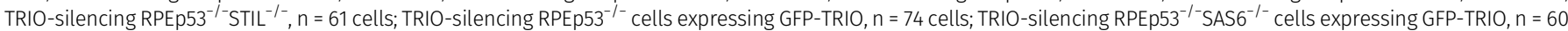

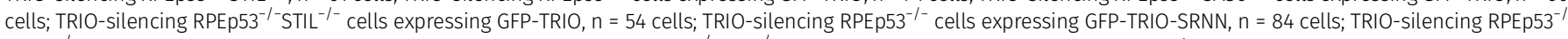
${ }^{-S A S 6}{ }^{-/-}$cells expressing GFP-TRIO-SRNN, $n=60$ cells; TRIO-silencing RPEp53 ${ }^{-/-}$STIL $^{-/-}$cells expressing GFP-TRIO-SRNN, $n=53$ cells). ${ }^{*} P<0.05 ;{ }^{* * *} P<0.001 ;$ NS, no significance. (c) Model showing the balance between centrosomal and acentrosomal microtubules during the control of Rac1 activation, FA dynamics, and directed cell migration. The centrosome acts as a controller and balances the formation of centrosomal and acentrosomal microtubules, which in turn restricts the random Rac1 activation caused by acentrosomal microtubules and activates Rac1 locally at cell front to induce membrane protrusion during directed cell migration. Interference with formation of the centrosome increases acentrosomal microtubules assembly, which results in the transportation of more TRIO protein, which in turn increases the excessive and random activation of Rac1. This then increases lamellipodia formation, leading to the loss of cell polarity and a negative impact on directed cell migration. 
culture dishes coated with $10 \mu \mathrm{g} / \mathrm{ml}$ fibronectin for $24 \mathrm{~h}$ at $50 \%$ confluence. Hypotonic shock was induced by a 3-min treatment with TEA-containing low ionic strength buffer ( $2.5 \mathrm{mM}$ triethanolamine [Sigma-Aldrich] in distilled water, pH 7.0). Cell bodies, including membrane-bound organelles, nuclei, cytoskeleton, and soluble cytoplasm, were removed by the use of a strong, pulsed hydrodynamic force using PBS containing protease inhibitors (Roche); this involved the use of a Waterpik (setting "3," Interplak dental water jet WJ6RW, Conair). Isolated FAs that remained bound to the dish were collected by scraping with a rubber policeman into 1× RIPA buffer (50 mM Tris-HCl, pH 8.0, 150 mM NaCl, 1.0\% NP-40, $0.5 \%$ sodium deoxycholate, and $0.1 \%$ SDS), and the resulting solution was sonicated for $15 \mathrm{~s}$ on ice. Protein concentrations were measured by the Bradford protein assay (Bio-Rad).

\section{Protein identification for LC-MS/MS analysis}

The precipitated FA protein pellets $(\sim 100 \mu \mathrm{g})$ were dissolved in resuspension buffer (6 M guanidine hydrochloride in $50 \mathrm{mM}$ ammonium bicarbonate), mixed with dithiothreitol (final concentration $10 \mathrm{mM}$; Sigma-Aldrich) at $37^{\circ} \mathrm{C}$ for $3 \mathrm{~h}$ for reduction, and then mixed with iodoacetamide (final concentration 10 mM; SigmaAldrich) at room temperature for $1 \mathrm{~h}$ in dark for alkylation. Subsequently, the buffer in the FA protein mixture was replaced with $50 \mathrm{mM}$ ammonium bicarbonate using a centrifugal filter device (Amicon Ultra-0.5, 10 kD; Millipore), then mixed with trypsin (Sequencing Grade; Promega) in a ratio of 1:50 (enzyme: protein mass ratio), and finally incubated at $37^{\circ} \mathrm{C}$ overnight. The trypsin digestion was terminated by adding formic acid (final concentration $0.5 \%, \mathrm{pH}$ $2 \sim 3)$. Finally, the mixture of digested peptides was desalted using a ZipTip C18 (Millipore), dried, and stored at $-80^{\circ} \mathrm{C}$.

For LC-MS/MS analysis, the dried digested peptides were dissolved in $0.1 \%$ formic acid and analyzed using an UltiMate 3000 RSLC nanoflow LC system (Thermo Fisher Scientific) interfaced to an Orbitrap Fusion Tribrid Mass Spectrometer (Thermo Fisher Scientific) equipped with a PicoView nanosprayer (New Objective). The peptides were loaded directly onto a $25-\mathrm{cm} \times 75-\mu \mathrm{m}$ C18 column (Acclaim PepMap RSLC; Thermo Fisher Scientific) and separated using a 40 -min linear gradient of $100 \%$ mobile phase A ( $0.1 \%$ formic acid in water) to $35 \%$ mobile phase $B$ (acetonitrile with $0.1 \%$ formic acid) at a flow rate of $500 \mathrm{nl} / \mathrm{min}$. The mass spectrometer was operated in the data-dependent acquisition using a 3-s duty cycle. In detail, full-scan MS spectra (MS1) were acquired using the Orbitrap ( $\mathrm{m} / \mathrm{z} 350-1,600)$ with the resolution in 60,000 at $\mathrm{m} / \mathrm{z} 400$ and automatic gain control target at $2 \times 10^{5}$, followed by quadrupole isolation of precursors at 2 Th width for CID MS/MS fragmentation and detection in the linear ion trap (automatic gain control target at $1 \times 10^{4}$ ) with previously selected ions dynamically excluded for $60 \mathrm{~s}$. Ions with single and an unrecognized charge state were also excluded. The LC-MS/MS raw files were normalized for label-free quantification by the software Progenesis QI for proteomics and searched using a Mascot Daemon 2.6.0 server. The mascot generic format (mgf) files were searched against the Swiss Prot (Swiss Institute of Bioinformatics) human database, carbamidomethyl (C) (variable), and oxidation (M) (variable). Up to two missed cleavages was allowed. The mass tolerance was set as $\pm 10 \mathrm{ppm}$ for the MS spectra and $\pm 0.6 \mathrm{D}$ for the MS/MS spectra. For peptide identification, the false discovery rate was adjusted to $1 \%$ or less.

\section{Microtubule isolation}

RPE cells were plated on culture dishes coated with $10 \mu \mathrm{g} / \mathrm{ml}$ fibronectin for $24 \mathrm{~h}$ at $50 \%$ confluence. The cells were then washed twice with PBS at $37^{\circ} \mathrm{C}$ and incubated with microtubule-stabilizing buffer (100 mM PIPES, pH 6.9, 5 mM MgCl 2 , mM EGTA, 2 M glycerol, $0.1 \%$ NP40, $10 \mathrm{mM} \beta$-glycerophosphate, $50 \mathrm{mM} \mathrm{NaF}, 0.3 \mu \mathrm{M}$ okadaic acid, and $1 \mathrm{mM}$ PMSF) containing protease inhibitors and phosphatase inhibitors (Roche) for $15 \mathrm{~min}$ at $37^{\circ} \mathrm{C}$. Cell lysates were collected by scraping with a rubber policeman and the suspension centrifuged at room temperature for $5 \mathrm{~min}$ at 1,000 $\mathrm{g}$. After centrifugation, the supernatants (soluble fraction) were collected and the pellets (insoluble fraction) were solubilized and sonicated in microtubule-stabilizing buffer for $15 \mathrm{~s}$ on ice.

\section{Rac1 activity assay}

Cells were lysed in $\mathrm{Mg}^{2+}$ lysis buffer (25 mM Hepes, pH 7.5, $150 \mathrm{mM}$ $\mathrm{NaCl}, 1 \%$ Triton $\mathrm{X}-100,10 \mathrm{mM} \mathrm{MgCl} 2,1 \mathrm{mM}$ EDTA, and 10\% glycerol) containing protease inhibitors and phosphatase inhibitors (Roche). The cell lysates were then incubated with GST-PAK-CRIB coupled to glutathione-Sepharose 4 beads using RAC1 pull-down activation assay biochem kit (Cytoskeleton, Inc.) for $1.5 \mathrm{~h}$ at $4^{\circ} \mathrm{C}$. The beads were washed in lysis buffer, re-suspended in SDS-PAGE sample buffer, and analyzed by Western blotting with anti-Rac1 antibody.

\section{Immunofluorescence analysis and image analysis}

For $\alpha$-tubulin/ $\gamma$-tubulin/DAPI and F-actin/ $\gamma$-tubulin staining, the cells were fixed with methanol at $-20^{\circ} \mathrm{C}$ for 20 min and blocked with blocking solution (3\% BSA/0.02\% Triton X-100 in PBS) at room temperature for $1 \mathrm{~h}$. Subsequently, the cells were incubated with the indicated primary antibodies in blocking solution at $4^{\circ} \mathrm{C}$ for $16 \mathrm{~h}$ and then incubated with fluorescent dye-conjugated secondary antibody at room temperature for $1 \mathrm{~h}$. For $\alpha$-tubulin/GOLPH2/DAPI staining, the cells were fixed with $4 \%$ paraformaldehyde at room temperature for $20 \mathrm{~min}$, permeabilized with PBS containing $0.01 \%$ Triton X-100 and $0.05 \%$ SDS at room temperature for $5 \mathrm{~min}$, and finally blocked with blocking solution ( $0.1 \%$ saponin and $0.2 \%$ BSA in PBS) at room temperature for $1 \mathrm{~h}$. Subsequently, the cells were incubated with the indicated primary antibodies in blocking solution at $4^{\circ} \mathrm{C}$ for $16 \mathrm{~h}$ and then incubated with fluorescent dyeconjugated secondary antibody at room temperature for $1 \mathrm{~h}$. For paxillin, $\alpha$-tubulin/DAPI, $\alpha$-tubulin/F-actin/DAPI, $\alpha$-tubulin/Factin/paxillin, and F-actin/cortactin staining, the cells were fixed with $4 \%$ paraformaldehyde in cytoskeleton buffer (10 mM MES pH 6.1, $138 \mathrm{mM} \mathrm{KCl}, 3 \mathrm{mM} \mathrm{MgCl}$, and $2 \mathrm{mM} \mathrm{EGTA)}$ at room temperature for $20 \mathrm{~min}$, permeabilized with cytoskeleton buffer containing $0.5 \%$ Triton X-100 at room temperature for $5 \mathrm{~min}$, and blocked with blocking solution (3\% BSA/0.02\% Triton $X-100$ in PBS) at room temperature for $60 \mathrm{~min}$. Subsequently, the cells were incubated with the indicated primary antibodies in blocking solution at $4^{\circ} \mathrm{C}$ for $16 \mathrm{~h}$ and then incubated with fluorescent dye-conjugated secondary antibody at room temperature for $1 \mathrm{~h}$. Finally, the cells were 
mounted on a magnetic chamber (Live Cell Instrument) and incubated with PBS containing N-propyl gallate for confocal, TIRF, or epi-fluorescence imaging.

Confocal images were obtained using an iLas multi-modal of TIRF (Roper)/spinning disk confocal (CSUX1, Yokogawa) microscope (Ti-E; Nikon) system equipped with $40 \times 1.3 \mathrm{NA}, 60 \times 1.49 \mathrm{NA}$, or $100 \times$ 1.49 NA Plan objective lens (Nikon) and a Coolsnap HQ2 CCD (Photometrics). TIRF images were obtained using the same microscope system and either a $60 \times 1.49$ NA or $100 \times 1.49$ NA plan objective lens (Nikon) on an Evolve EMCCD (Photometrics) with an 100-nm evanescent field depth. All confocal and TIRF images were captured and processed using Metamorph software. Epifluorescence images were obtained using a microscope (DMRBE; Leica) coupled with a $63 \times$ NA 1.4 objective lens (Leica) and an 512B EMCCD (Andor) operated by Micro-Manager 1.4 software (Leica), or an epi-fluorescence microscope system (Ti-E; Nikon) coupled with a $60 \times$ NA 1.49 plan objective lens (Nikon) and an sCMOS camera (OHCA-Flash 4.0, 1,024 × 1,024 pixels; Hamamatsu) operated by NISElements software (Nikon).

To determine the FA area, TIRF images of paxillin-stained cells were thresholded to highlight only the FAs and the areas of the regions recorded using Metamorph. The area of recorded FAs was organized to give the adhesion size distribution. The results are presented graphically using Excel software (Microsoft).

\section{Time-lapse microscopy and image analysis}

To analyze the dynamics of paxillin, cells expressing mApplepaxillin were mounted on a magnetic chamber (Live Cell Instrument) and incubated in phenol red-free culture medium with $25 \mathrm{mM}$ Hepes $(\mathrm{pH}=7.4)$ and imaged by TIRF using a $100 \times 1.49 \mathrm{NA}$ objective with an evanescent field depth of $\sim 100 \mathrm{~nm}$ on the microscope system described above. Stage temperature was maintained at $37^{\circ} \mathrm{C}$ with an airstream incubator (Nevtek) and focus was maintained using the PerfectFocus system (Nikon). TIRF images of mApple-paxillin were captured at 1-min intervals using an Evolve EMCCD (Photometrics). To analyze the intensity changes of mApplepaxillin during FA turnover, the area of single FAs was hand-outlined in the paxillin channel over time. The integrated intensities within the areas and backgrounds around the areas were recorded for each time point. The background-subtracted, photobleach-corrected, and normalized (to the max value) intensity values were plotted as a function of time to determine when the intensity increases as the FA initiated and when the intensity decreases during termination. Thus, the duration of FA assembly, FA maturation, and FA disassembly was determined.

To analyze the dynamics of microtubules, cells expressing GFPEB3 were mounted on a magnetic chamber (Live Cell Instrument) and incubated in phenol red-free culture medium with $25 \mathrm{mM}$ Hepes $(\mathrm{pH}=7.4$ ) and imaged by spinning disk confocal using a $100 \times$ 1.49 NA objective on the microscope system described above. Confocal images of GFP-EB3 were captured at 2-s intervals using an EMCCD (ProEM; Princeton). To track the dynamics of GFP-EB3, a microtubule plus end-tracking program (Applegate et al, 2011) was used to obtained microtubule growth speed and growth lifetime.

To image the recruitment of TRIO in FAs, cells expressing mApple-paxillin together with GFP-TRIO or GFP-TRIO-SRNN were mounted on a magnetic chamber (Live Cell Instrument) and incubated in phenol red-free medium with $25 \mathrm{mM}$ Hepes $(\mathrm{pH}=7.4)$ and imaged by TIRF using a $100 \times 1.49$ NA objective with an evanescent field depth of $\sim 100 \mathrm{~nm}$ on the microscope system that has been described above.

\section{FRET (Förster resonance energy transfer) analysis}

Cells expressing FRET-based Rac1 biosensors (pTriEx4-Rac1-2G) were mounted on a magnetic chamber (Live Cell Instrument) and incubated in phenol red-free culture medium with $25 \mathrm{mM}$ Hepes ( $\mathrm{pH}=7.4)$. The images were obtained by an epi-fluorescence microscope system (Ti-E; Nikon) equipped with a $40 \times 1.30 \mathrm{NA}$ objective lens (Nikon) and an ORCA-Flash4.0 V2 Digital CMOS camera (Hamamatsu) operated by NIS-Elements software (Nikon) with a 420DF20 excitation filter and two emission filters controlled by a filter changer (475DF40 for CFP and 535DF25 for YFP). The CFP images were collected using a 420DF20 excitation filter and a 475DF40 emission filter; the FRET images were collected using a 420DF20 excitation filter and a 535DF25 emission filter. Pixel-bypixel analysis of the FRET/CFP ratio images was carried out based on background-subtracted fluorescence intensity images of the CFP and FRET images using Matlab (The MathWorks).

\section{Cell spreading assay and image analysis}

Cells growing on tissue culture plates were trypsinized and reseeded on coverslips coated with $10 \mu \mathrm{g} / \mathrm{ml}$ fibronectin for $30 \mathrm{~min}$ to allow them to adhere and spread. Next, the cells were fixed with $4 \%$ paraformaldehyde in PBS for 20 min at room temperature and then imaged using a microscope (Eclipse TS100; Nikon) coupled with a $20 \times 0.45$ NA objective lens (Nikon) and a WHITE CCD camera operated by ISCapture software (TUCSEN). To calculate the cell spreading area, the cell area was manually circled on the phase images using Metamorph image analysis software (Molecular Device) and the results are presented graphically using Excel software (Microsoft).

\section{Adhesion assay}

The cell adhesion assays used 96 -well plates that had been pretreated with $1 \%$ denatured $\mathrm{BSA}$ at $37^{\circ} \mathrm{C}$ for $1 \mathrm{~h}$ and then coated with $10 \mu \mathrm{g} / \mathrm{ml}$ fibronectin. Cells growing on tissue culture plates were trypsinized, re-suspended in serum-free medium, and then reseeded on the fibronectin-coated 96-well plates for $30 \mathrm{~min}$ or overnight ( 16 h). After incubation, any non-attached cells were removed completely by washing with PBS twice and the adherent cells were fixed with $5 \%$ glutaraldehyde in $\mathrm{H}_{2} \mathrm{O}$ for 25 min at room temperature; this was followed by staining with $0.1 \%$ crystal violet in $\mathrm{H}_{2} \mathrm{O}$ for $25 \mathrm{~min}$ at room temperature. After removing any unbound crystal violet, the crystal violet-labeled adherent cells were solubilized in $50 \mu$ solution $\mathrm{A}$ ( $50 \%$ ethanol and $0.1 \%$ acetic acid in $\mathrm{H}_{2} \mathrm{O}$ ) and the amount of crystal violet present measured using a Thermo Fisher Scientific Multiskan Spectrum at OD $550 \mathrm{~nm}$. The results are presented graphically using Excel software (Microsoft). 


\section{Wound-healing assay}

Cells were growing on a chamber (with $500 \mu \mathrm{m}$ space; SPL) embedded in $10 \mu \mathrm{g} / \mathrm{ml}$ fibronectin-coated 12-well plates in the culture medium for $16 \mathrm{~h}$ to create a wound in the confluent cells, and the plates were then placed in a temperature $/ \mathrm{CO}_{2}$-controlled chamber on a microscope (Axio Observer.Z1; Zeiss) equipped with a $10 \times 0.25$ NA objective lens (Zeiss). Time-lapse images were obtained at 15-min intervals over $12 \mathrm{~h}$ using a Rolera EM- $C^{2}$ EMCCD camera operated by Zen image analysis software (Zeiss). To calculate cell migration parameters, the centers of cell nuclei of cells at wound edge were manually tracked and positions recorded from the timelapse image series using the "track points" function in Metamorph. Position and time data were then transferred to Excel to calculate the migration parameters, including cell migration speed and directional persistence. Speed was calculated as the total length of the migration path divided by the duration of migration. Directional persistence was calculated as the net migration distance divided by the total length of the migration path.

\section{Statistical analysis}

Statistical significance was measured by either the $t$ test or the ANOVA test.

\section{Supplementary Information}

Supplementary Information is available at https://doi.org/10.26508/lsa. 201800135.

\section{Acknowledgements}

We thank the Academia Sinica Common Mass Spectrometry Facilities (Institute of Biological Chemistry, Academia Sinica, Taipei, Taiwan) for their help with the proteomics data analysis; Prof. Won-Jing Wang (National Yang-Ming University, Taipei, Taiwan) for providing the RPEp53 ${ }^{-1-}, \mathrm{RPEp}^{-1 /} \mathrm{SAS6}^{-1-}$, and RPEp53 ${ }^{-/-} \mathrm{STIL}^{-1-}$ cells; and Prof. Anne Debant (University of Montpellier, Montpellier, France) for providing the GFP-TRIO and GFP-TRIO-SRNN constructs. J-C Kuo is supported by research grants from the Taiwan Ministry of Science and Technology (MOST 103-2628-B-010-003-MY4; MOST 106-2633-B010-002-; MOST 107-2633-B-010-001-; MOST 107-2320-B-010-049-), the Novel Bioengineering and Technological Approaches to Solve Two Major Health Problems in Taiwan sponsored by the Taiwan Ministry of Science and Technology Academic Excellence Program (MOST 107-2633-B-009-003), Cancer Progression Research Center (National Yang-Ming University) from The Featured Areas Research Center Program within the framework of the Higher Education Sprout Project by the Ministry of Education in Taiwan, the Yen Tjing Ling Medical Foundation, and the Ministry of Education's “Aim for the Top University Plan."

\section{Author Contributions}

H-W Cheng: data curation, formal analysis, validation, and methodology.

C-T Hsiao: data curation, formal analysis, validation, and methodology.

Y-Q Chen: data curation, formal analysis, and methodology.
C-M Huang: data curation and formal analysis.

S-I Chan: data curation and formal analysis.

A Chiou: methodology and writing-review and editing.

J-C Kuo: conceptualization, data curation, formal analysis, supervision, funding acquisition, validation, investigation, project administration, writing-original draft, review, and editing.

\section{Conflict of Interest Statement}

The authors declare that they have no conflict of interest.

\section{References}

Applegate KT, Besson S, Matov A, Bagonis MH, Jaqaman K, Danuser G (2011) plusTipTracker: Quantitative image analysis software for the measurement of microtubule dynamics. J Struct Biol 176: 168-184. doi:10.1016/j.jsb.2011.07.009

Arquint C, Nigg EA (2016) The PLK4-STIL-SAS-6 module at the core of centriole duplication. Biochem Soc Trans 44: 1253-1263. doi:10.1042/bst20160116

Bateman J, Shu H, Van Vactor D (2000) The guanine nucleotide exchange factor trio mediates axonal development in the Drosophila embryo. Neuron 26: 93-106. doi:10.1016/s0896-6273(00)81141-1

Bazzi H, Anderson KV (2014) Acentriolar mitosis activates a p53-dependent apoptosis pathway in the mouse embryo. Proc Natl Acad Sci U S A 111: E1491-E1500. doi:10.1073/pnas.1400568111

Bellanger JM, Astier C, Sardet C, Ohta Y, Stossel TP, Debant A (2000) The Rac1and RhoG-specific GEF domain of Trio targets filamin to remodel cytoskeletal actin. Nat Cell Biol 2: 888-892. doi:10.1038/35046533

Bellanger JM, Lazaro JB, Diriong S, Fernandez A, Lamb N, Debant A (1998) The two guanine nucleotide exchange factor domains of Trio link the Rac1 and the RhoA pathways in vivo. Oncogene 16: 147-152. doi:10.1038/sj. onc.1201532

Bertling E, Hotulainen P, Mattila PK, Matilainen T, Salminen M, Lappalainen P (2004) Cyclase-associated protein 1 (CAP1) promotes cofilin-induced actin dynamics in mammalian nonmuscle cells. Mol Biol Cell 15: 2324-2334. doi:10.1091/mbc.e04-01-0048

Burridge K, Fath K, Kelly T, Nuckolls G, Turner C (1988) Focal adhesions: Transmembrane junctions between the extracellular matrix and the cytoskeleton. Annu Rev Cell Biol 4: 487-525. doi:10.1146/annurev. cellbio.4.1.487

Chabin-Brion K, Marceiller J, Perez F, Settegrana C, Drechou A, Durand G, Pous C (2001) The Golgi complex is a microtubule-organizing organelle. Mol Biol Cell 12: 2047-2060. doi:10.1091/mbc.12.7.2047

Chang YC, Nalbant P, Birkenfeld J, Chang ZF, Bokoch GM (2008) GEF-H1 couples nocodazole-induced microtubule disassembly to cell contractility via RhoA. Mol Biol Cell 19: 2147-2153. doi:10.1091/mbc.e0712-1269

Chao WT, Kunz J (2009) Focal adhesion disassembly requires clathrindependent endocytosis of integrins. FEBS Lett 583: 1337-1343. doi:10.1016/j.febslet.2009.03.037

Chen HY, Wu CT, Tang CC, Lin YN, Wang WJ, Tang TK (2017) Human microcephaly protein RTTN interacts with STIL and is required to build full-length centrioles. Nat Commun 8: 247. doi:10.1038/s41467-01700305-0

David A, Liu F, Tibelius A, Vulprecht J, Wald D, Rothermel U, Ohana R, Seitel A Metzger J, Ashery-Padan R, et al (2014) Lack of centrioles and primary cilia in STIL(-/-) mouse embryos. Cell Cycle 13: 2859-2868. doi:10.4161/ 15384101.2014.946830 
Debant A, Serra-Pages C, Seipel K, O'Brien S, Tang M, Park SH, Streuli M (1996) The multidomain protein Trio binds the LAR transmembrane tyrosine phosphatase, contains a protein kinase domain, and has separate rac-specific and rho-specific guanine nucleotide exchange factor domains. Proc Natl Acad Sci U S A 93: 5466-5471. doi:10.1073/ pnas.93.11.5466

Del Pozo MA, Kiosses WB, Alderson NB, Meller N, Hahn KM, Schwartz MA (2002) Integrins regulate GTP-Rac localized effector interactions through dissociation of Rho-GDI. Nat Cell Biol 4: 232-239. doi:10.1038/ncb759

Echarri A, Del Pozo MA (2006) Caveolae internalization regulates integrindependent signaling pathways. Cell Cycle 5: 2179-2182. doi:10.4161/ cc.5.19.3264

Efimov A, Kharitonov A, Efimova N, Loncarek J, Miller PM, Andreyeva N, Gleeson P, Galjart N, Maia AR, McLeod IX, et al (2007) Asymmetric CLASP-dependent nucleation of noncentrosomal microtubules at the trans-Golgi network. Dev Cell 12: 917-930. doi:10.1016/j. devcel.2007.04.002

Etienne-Manneville S, Hall A (2001) Integrin-mediated activation of Cdc42 controls cell polarity in migrating astrocytes through PKCzeta. Cell 106: 489-498. doi:10.1016/s0092-8674(01)00471-8

Ezratty EJ, Bertaux C, Marcantonio EE, Gundersen GG (2009) Clathrin mediates integrin endocytosis for focal adhesion disassembly in migrating cells. J Cell Biol 187: 733-747. doi:10.1083/jcb.200904054

Ezratty EJ, Partridge MA, Gundersen GG (2005) Microtubule-induced focal adhesion disassembly is mediated by dynamin and focal adhesion kinase. Nat Cell Biol 7: 581-590. doi:10.1038/ncb1262

Franz CM, Jones GE, Ridley AJ (2002) Cell migration in development and disease. Dev Cell 2: 153-158. doi:10.1016/s1534-5807(02)00120-x

Fritz RD, Menshykau D, Martin K, Reimann A, Pontelli V, Pertz O (2015) SrGAP2Dependent integration of membrane geometry and Slit-RoboRepulsive cues regulates fibroblast contact inhibition of locomotion. Dev Cell 35: 78-92. doi:10.1016/j.devcel.2015.09.002

Gao Y, Xing J, Streuli M, Leto TL, Zheng Y (2001) Trp(56) of rac1 specifies interaction with a subset of guanine nucleotide exchange factors. J Biol Chem 276: 47530-47541. doi:10.1074/jbc.m108865200

Gupton SL, Waterman-Storer CM (2006) Spatiotemporal feedback between actomyosin and focal-adhesion systems optimizes rapid cell migration. Cell 125: 1361-1374. doi:10.1016/j.cell.2006.05.029

Henty-Ridilla JL, Rankova A, Eskin JA, Kenny K, Goode BL (2016) Accelerated actin filament polymerization from microtubule plus ends. Science 352: 1004-1009. doi:10.1126/science.aaf1709

Huttenlocher A, Ginsberg MH, Horwitz AF (1996) Modulation of cell migration by integrin-mediated cytoskeletal linkages and ligand-binding affinity. J Cell Biol 134: 1551-1562. doi:10.1083/jcb.134.6.1551

Hynes RO (2002) Integrins: Bidirectional, allosteric signaling machines. Cell 110: 673-687. doi:10.1016/s0092-8674(02)00971-6

Innocenti M, Tenca P, Frittoli E, Faretta M, Tocchetti A, Di Fiore PP, Scita G (2002) Mechanisms through which Sos-1 coordinates the activation of Ras and rac. J Cell Biol 156: 125-136. doi:10.1083/jcb.200108035

Jockusch BM, Bubeck P, Giehl K, Kroemker M, Moschner J, Rothkegel M, Rudiger M, Schluter K, Stanke G, Winkler J (1995) The molecular architecture of focal adhesions. Annu Rev Cell Dev Biol 11: 379-416. doi:10.1146/annurev.cb.11.110195.002115

Kim M, Fong CS, Tsou MF (2014) Centriole duplication: When PLK4 meets Ana2/STIL. Curr Biol 24: R1046-R1048. doi:10.1016/j.cub.2014.09.079

Koonce MP, Cloney RA, Berns MW (1984) Laser irradiation of centrosomes in newt eosinophils: Evidence of centriole role in motility. I Cell Biol 98: 1999-2010. doi:10.1083/jcb.98.6.1999

Kuo JC, Han X, Hsiao CT, Yates 3rd JR, Waterman CM (2011) Analysis of the myosin-II-responsive focal adhesion proteome reveals a role for beta-Pix in negative regulation of focal adhesion maturation. Nat Cell Biol 13: 383-393. doi:10.1038/ncb2216
Kuo JC, Han X, Yates 3rd JR, Waterman CM (2012) Isolation of focal adhesion proteins for biochemical and proteomic analysis. Methods Mol Biol 757: 297-323. doi:10.1007/978-1-61779-166-6_19

Lauffenburger DA, Horwitz AF (1996) Cell migration: A physically integrated molecular process. Cell 84: 359-369. doi:10.1016/s0092-8674(00)81280-5

Leidel S, Delattre M, Cerutti L, Baumer K, Gonczy P (2005) SAS-6 defines a protein family required for centrosome duplication in C. elegans and in human cells. Nat Cell Biol 7: 115-125. doi:10.1038/ ncb1220

Matov A, Applegate K, Kumar P, Thoma C, Krek W, Danuser G, Wittmann T (2010) Analysis of microtubule dynamic instability using a plus-end growth marker. Nat Methods 7: 761-768. doi:10.1038/nmeth.1493

Medley QG, Buchbinder EG, Tachibana K, Ngo H, Serra-Pages C, Streuli M (2003) Signaling between focal adhesion kinase and trio. J Biol Chem 278: 13265-13270. doi:10.1074/jbc.m300277200

Medley QG, Serra-Pages C, Iannotti E, Seipel K, Tang M, O'Brien SP, Streuli M (2000) The trio guanine nucleotide exchange factor is a RhoA target. Binding of RhoA to the trio immunoglobulin-like domain. J Biol Chem 275: 36116-36123. doi:10.1074/jbc.m003775200

Miki H, Yamaguchi H, Suetsugu S, Takenawa T (2000) IRSp53 is an essential intermediate between Rac and WAVE in the regulation of membrane ruffling. Nature 408: 732-735. doi:10.1038/35047107

Mouneimne G, Hansen SD, Selfors LM, Petrak L, Hickey MM, Gallegos LL, Simpson KJ, Lim J, Gertler FB, Hartwig JH, et al (2012) Differential remodeling of actin cytoskeleton architecture by profilin isoforms leads to distinct effects on cell migration and invasion. Cancer Cell 22: 615-630. doi:10.1016/j.ccr.2012.09.027

Newsome TP, Schmidt S, Dietzl G, Keleman K, Asling B, Debant A, Dickson BJ (2000) Trio combines with dock to regulate Pak activity during photoreceptor axon pathfinding in Drosophila. Cell 101: 283-294. doi:10.1016/s0092-8674(00)80838-7

Nishimura T, Kaibuchi K (2007) Numb controls integrin endocytosis for directional cell migration with aPKC and PAR-3. Dev Cell 13: 15-28. doi:10.1016/j.devcel.2007.05.003

Nobes CD, Hall A (1999) Rho GTPases control polarity, protrusion, and adhesion during cell movement. J Cell Biol 144: 1235-1244. doi:10.1083/ jcb.144.6.1235

Oser M, Condeelis J (2009) The cofilin activity cycle in lamellipodia and invadopodia. J Cell Biochem 108: 1252-1262. doi:10.1002/ jcb.22372

Portran D, Schaedel L, Xu Z, Thery M, Nachury MV (2017) Tubulin acetylation protects long-lived microtubules against mechanical ageing. Nat Cell Biol 19: 391-398. doi:10.1038/ncb3481

Ridley AJ, Schwartz MA, Burridge K, Firtel RA, Ginsberg MH, Borisy G, Parsons JT, Horwitz AR (2003) Cell migration: Integrating signals from front to back. Science 302: 1704-1709. doi:10.1126/science.1092053

Rooney C, White G, Nazgiewicz A, Woodcock SA, Anderson KI, Ballestrem C, Malliri A (2010) The Rac activator STEF (Tiam2) regulates cell migration by microtubule-mediated focal adhesion disassembly. EMBO Rep 11: 292-298. doi:10.1038/embor.2010.10

Sanz-Moreno V, Gadea G, Ahn J, Paterson H, Marra P, Pinner S, Sahai E, Marshall CJ (2008) Rac activation and inactivation control plasticity of tumor cell movement. Cell 135: 510-523. doi:10.1016/j.cell.2008.09.043

Schwartz MA, Schaller MD, Ginsberg MH (1995) Integrins: Emerging paradigms of signal transduction. Annu Rev Cell Dev Biol 11: 549-599. doi:10.1146/ annurev.cellbio.11.1.549

Sloboda RD (2015) Isolation of microtubules and microtubule-associated proteins using Paclitaxel. Cold Spring Harb Protoc 2015: pdb prot081190. doi:10.1101/pdb.prot081190

Stehbens S, Wittmann T (2012) Targeting and transport: How microtubules control focal adhesion dynamics. J Cell Biol 198: 481-489. doi:10.1083/ jcb.201206050 
Tsygankov D, Bilancia CG, Vitriol EA, Hahn KM, Peifer M, Elston TC (2014) CellGeo: A computational platform for the analysis of shape changes in cells with complex geometries. J Cell Biol 204: 443-460. doi:10.1083/ jcb.201306067

van Haren J, Boudeau J, Schmidt S, Basu S, Liu Z, Lammers D, Demmers J, Benhari J, Grosveld F, Debant A, et al (2014) Dynamic microtubules catalyze formation of navigator-TRIO complexes to regulate neurite extension. Curr Biol 24: 1778-1785. doi:10.1016/j. cub.2014.06.037

van Rijssel J, Hoogenboezem M, Wester L, Hordijk PL, Van Buul JD (2012) The $\mathrm{N}$-terminal $\mathrm{DH}-\mathrm{PH}$ domain of Trio induces cell spreading and migration by regulating lamellipodia dynamics in a Rac1-dependent fashion. PLoS One 7: e29912. doi:10.1371/journal.pone.0029912

Vinogradova T, Paul R, Grimaldi AD, Loncarek J, Miller PM, Yampolsky D, Magidson V, Khodjakov A, Mogilner A, Kaverina I (2012) Concerted effort of centrosomal and Golgi-derived microtubules is required for proper Golgi complex assembly but not for maintenance. Mol Biol Cell 23: 820-833. doi:10.1091/mbc.e11-06-0550

Wakida NM, Botvinick EL, Lin J, Berns MW (2010) An intact centrosome is required for the maintenance of polarization during directional cell migration. PLoS One 5: e15462. doi:10.1371/journal.pone.0015462

Wang WJ, Acehan D, Kao CH, Jane WN, Uryu K, Tsou MF (2015) De novo centriole formation in human cells is error-prone and does not require SAS-6 self-assembly. Elife 4. doi:10.7554/elife.10586

Waterman-Storer CM, Worthylake RA, Liu BP, Burridge K, Salmon ED (1999) Microtubule growth activates Rac1 to promote lamellipodial protrusion in fibroblasts. Nat Cell Biol 1: 45-50. doi:10.1038/9018

Webb DJ, Parsons JT, Horwitz AF (2002) Adhesion assembly, disassembly and turnover in migrating cells: Over and over and over again. Nat Cell Biol 4: E97-E100. doi:10.1038/ncb0402-e97
Wittmann T, Bokoch GM, Waterman-Storer CM (2003) Regulation of leading edge microtubule and actin dynamics downstream of Rac1. I Cell Biol 161: 845-851. doi:10.1083/jcb.200303082

Wu J, de Heus C, Liu Q, Bouchet BP, Noordstra I, Jiang K, Hua S, Martin M, Yang C, Grigoriev I, et al (2016) Molecular pathway of microtubule organization at the Golgi apparatus. Dev Cell 39: 44-60. doi:10.1016/j. devcel.2016.08.009

Wu JC, Chen YC, Kuo CT, Wenshin Yu H, Chen YQ, Chiou A, Kuo JC (2015) Focal adhesion kinase-dependent focal adhesion recruitment of $\mathrm{SH} 2$ domains directs SRC into focal adhesions to regulate cell adhesion and migration. Sci Rep 5: 18476. doi:10.1038/srep18476

Yu HW, Chen YQ, Huang CM, Liu CY, Chiou A, Wang YK, Tang MJ, Kuo JC (2015) beta-PIX controls intracellular viscoelasticity to regulate lung cancer cell migration. J Cell Mol Med 19: 934-947. doi:10.1111/jcmm.12441

Zaidel-Bar R, Geiger B (2010) The switchable integrin adhesome. J Cell Sci 123: 1385-1388. doi:10.1242/jcs.066183

Zaidel-Bar R, Itzkovitz S, Ma'ayan A, Iyengar R, Geiger B (2007) Functional atlas of the integrin adhesome. Nat Cell Biol 9: 858-867. doi:10.1038/ ncb0807-858

Zhang J, Wang YL (2017) Centrosome defines the rear of cells during mesenchymal migration. Mol Biol Cell 28: 3240-3251. doi:10.1091/mbc. e17-06-0366

Zhu X, Kaverina I (2013) Golgi as an MTOC: Making microtubules for its own good. Histochem Cell Biol 140: 361-367. doi:10.1007/s00418-013-1119-4

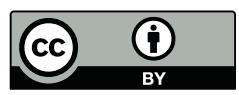

License: This article is available under a Creative Commons License (Attribution 4.0 International, as described at https://creativecommons.org/ licenses/by/4.0/). 